\title{
Monitoring Protein-RNA Interaction Dynamics in vivo at High Temporal Resolution using XCRAC
}

Stuart W. McKellar ${ }^{1}$, Ivayla Ivanova ${ }^{1}$, Robert W. van Nues ${ }^{2}$, Ross A. Cordiner ${ }^{3}$, Mehak Chauhan ${ }^{1}$, Niki Christopoulou ${ }^{1}$, Will Worboys ${ }^{4}$, Andrew Langford ${ }^{4}$, Torben Heick Jensen ${ }^{3}$, Sander Granneman ${ }^{1}$

${ }^{1}$ Centre for Engineering Biology, University of Edinburgh ${ }^{2}$ Institute of Cell Biology, University of Edinburgh ${ }^{3}$ Department of Molecular Biology and Genetics, Aarhus University ${ }^{4}$ UVO3 Ltd.

\section{Corresponding Author}

Sander Granneman

sander.granneman@ed.ac.uk

\section{Citation}

McKellar, S.W., Ivanova, I., van

Nues, R.W., Cordiner, R.A.,

Chauhan, M., Christopoulou, N.,

Worboys, W., Langford, A., Jensen, T.H.,

Granneman, S. Monitoring Protein-

RNA Interaction Dynamics in vivo

at High Temporal Resolution using

XCRAC. J. Vis. Exp. (159), e61027,

doi:10.3791/61027 (2020).

\section{Date Published}

May 9, 2020

\section{DOI}

$10.3791 / 61027$

URL

jove.com/video/61027

\section{Abstract}

The interaction between RNA-binding proteins (RBPs) and their RNA substrates exhibits fluidity and complexity. Within its lifespan, a single RNA can be bound by many different RBPs that will regulate its production, stability, activity, and degradation. As such, much has been done to understand the dynamics that exist between these two types of molecules. A particularly important breakthrough came with the emergence of 'cross-linking and immunoprecipitation' (CLIP). This technique allowed stringent investigation into which RNAs are bound by a particular RBP. In short, the protein of interest is UV cross-linked to its RNA substrates in vivo, purified under highly stringent conditions, and then the RNAs covalently cross-linked to the protein are converted into cDNA libraries and sequenced. Since its conception, many derivative techniques have been developed in order to make CLIP amenable to particular fields of study. However, cross-linking using ultraviolet light is notoriously inefficient. This results in extended exposure times that make the temporal study of RBP-RNA interactions impossible. To overcome this issue, we recently designed and built much-improved UV irradiation and cell harvesting devices. Using these new tools, we developed a protocol for time-resolved analyses of RBP-RNA interactions in living cells at high temporal

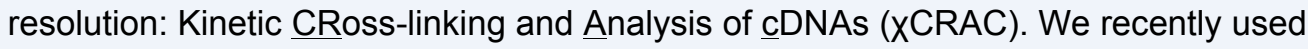
this technique to study the role of yeast RBPs in nutrient stress adaptation. This manuscript provides a detailed overview of the XCRAC method and presents recent results obtained with the Nrd1 RBP.

\section{Introduction}

RNAs often rely on RBPs to exert their function, which has led to great interest in understanding the dynamics between these molecules. Many RBPs have been identified in a wide variety of organisms. However, it has always been notoriously difficult to study RBP-RNA interactions in vivo. A major breakthrough in studying such interactions 
came with the emergence of CLIP ${ }^{1}$. This method utilizes ultraviolet (UV, $254 \mathrm{~nm}$ ) irradiation to induce covalent bonds between RBPs and their directly bound RNAs (i.e., zerodistance cross-linking). Subsequently, the RBP of interest is immunopurified under stringent conditions to ensure that only RNAs covalently cross-linked to the proteins are identified. Bound RNAs are then partially digested with RNases and subsequently converted into cDNA libraries for sequencing. The high purification stringency is important as it greatly increases the specificity of protein and RNA recovery, which is also further enhanced through SDS-PAGE purification of the cross-linked ribonucleoprotein (RNP) complex. CLIP and related methods also provide nucleotide resolution insight into the protein binding site, because during the preparation of the sequencing library, amino acids that cross-linked to the RNA frequently terminate the reverse transcriptase or cause the enzyme to introduce mutations at this site ${ }^{1,2,3}$.

Since its introduction, the original CLIP protocol has produced a remarkable variety of derivative methodologies. A particularly important breakthrough came with the development of HITS-CLIP (or CLIP-seq), which merges high-throughput sequencing with the CLIP approach ${ }^{3}$. This has since been adopted by all CLIP-based methodologies. iCLIP introduced improvements in the RNase-mediated trimming and adaptor ligation techniques that facilitate more accurate mapping of the RBP binding sites ${ }^{4}$. PAR-CLIP combined 4thio-uridine/uracil labeling with cross-linking at $365 \mathrm{~nm}$, making it possible to map cross-linking sites by analyzing T-C substitutions ${ }^{5}$. CRAC, urea-iCLIP, dCLIP, and uvCLAP introduced denaturing conditions and dual affinity purification steps that further reduce background binding to the affinity resin and further increase the specificity of protein capture $^{2,6,7,8,9}$. Additionally, CRAC, uvCLAP, and dCLIP introduced tagging the RBP of interest with an affinity tag, thus overcoming the need to generate specific antibodies.

Several optimizations have also been made to expedite the CLIP methodology. The original CLIP protocol utilized radiolabeling of the captured RNAs in order to visualize the RBP-RNA complexes after SDS-PAGE. However, the use of radioactivity can be problematic for laboratories not set up for such work. irCLIP incorporates a fluorophorecoupled adaptor that facilitates visualization through infrared imaging $^{10}$ and sCLIP utilizes biotinylation of captured RNAs in order to visualize them through streptavidinconjugated HRP ${ }^{11}$. Furthermore, eCLIP completely forgoes RNA labeling; instead the protein is excised based solely on its known size ${ }^{12}$. Streptavidin-based purification has also been used to speed up the process of library preparation in FAST-iCLIP, where a biotinylated 3' adaptor is ligated onto the RNAs and used to enable purification after reverse transcription and circularization ${ }^{13}$. Additional enhancements to the iCLIP protocol also greatly increased the complexity of the libraries ${ }^{4}$.

Finally, CLIP has been modified to enable capture of RBPs from different cellular subcompartments ${ }^{14,15}$, to visualize newly transcribed RNAs using pulsed induction of photoactivable ribonucleosides $5,16,17$, to capture methylated RNAs ${ }^{18,19,20}$, to examine RNA-RNA interactions ${ }^{21,22}$, and to map $3^{\prime}$ ends ${ }^{23,24}$.

Despite the great contributions of CLIP-based techniques in aiding our understanding of the interactions between RBPs and RNAs, it has been limited by the inefficiency of UV cross-linking. Although culture cells grown in a monolayer are generally relatively easy to cross-link, this is significantly more challenging in tissues or cells in solution. Tissues can require multiple rounds of UV exposure in order to penetrate 
to the required cell layers, while microbial cells are often grown in rich mediums that contain aromatic, UV-absorbing compounds ${ }^{25}$. Indeed, UV irradiation times of up to $30 \mathrm{~min}$ have been used to generate sufficient cross-linking between RBPs and their bound RNAs for such samples $26,27,28$. This extended UV exposure induces stress responses within the cell, such UV-induced DNA damage, which can contaminate the final data in some applications.

The majority of CLIP studies have focused on generating single "snapshots" of specific protein-RNA interactions in a cell. However, protein-RNA interactions are inherently dynamic, particularly when cells are subject to changes in their environment. This can include a sudden reduction in the availability of essential nutrients or rapid changes in temperature. As such, to truly understand the role of an RBP during stress, it is best to perform time-resolved analyses because they can capture the full spectrum of RBP targets during stress and determine at what stage of the stress response the chosen RBP is active. In particular, studies in yeast showed that the first few minutes of adaptation are absolutely crucial for survival and RNA half-lives in bacteria can vary from minutes to seconds $29,30,31,32,33$. Therefore, such time-resolved analyses should ideally be performed at high temporal resolution. However, the long cross-linking times make the study of early stage adaptive responses particularly challenging.

In order to overcome these issues, we recently developed an improved method that is capable of cross-linking and harvesting cells on minute-long timescales. Our XCRAC method allows quantitative measurement of dynamic changes in RBP-RNA interactions at previously unwitnessed resolution. Crucial to this method was the development of a novel UV irradiation device ${ }^{32}$ that reduces the required cross- linking time in yeast and bacteria in solution around 10-fold, effectively freezing RBP-RNA interactions instantaneously. In addition, in order to rapidly harvest the cells after UV irradiation, we developed a vacuum filtration device that can harvest exponentially growing yeast in a $0.5 \mathrm{~L}$ culture in around $30 \mathrm{~s}^{32}$. These technological innovations allow the study of RBP-RNA dynamics at minute-scale resolution. Additionally, we also introduced several optimizations to the original CRAC protocol ${ }^{2}$ in order to increase its practicality.

Using XCRAC, we recently studied the targetome of a yeast nuclear RBP, Nab3, in response to glucose deprivation. In Saccharomyces cerevisiae, Nab3 can form a complex with Nrd1, an RBP, and the RNA helicase Sen1 to form the NNS complex. NNS binding to the RNA polymerase and the nascent transcript can trigger transcriptional termination ${ }^{34}$. This complex is mostly involved in removing cryptic noncoding RNA transcripts but has also been shown to regulate expression of protein-coding genes. The study showed differential targeting of Nab3 to noncoding and coding transcripts after only a minute of stress ${ }^{32}$. We demonstrated that co-transcriptional termination by Nab3 results in a very transient, pulse-like expression of retrotransposon genes, which would have been difficult to detect using traditional CLIP-based approaches. Additionally, the short UV irradiation times in our UV cross-linker also significantly increased the recovery of short-lived noncoding $\mathrm{RNAs}^{32}$. XCRAC will likely be a crucial tool in elucidating not only how RBPs shape the response to stress on immediate timescales but also their changing roles during the whole lifecycle of a response. This manuscript provides a detailed overview of all the steps in the $X$ CRAC protocol. For illustrative purposes, the method was used to study the yeast Nrd1 protein, which is involved in transcriptional termination and RNA decay ${ }^{35,36}$, and its RNA targetome in response to glucose deprivation across a 
multitude of timepoints. Finally, we also demonstrate that our

UV irradiation unit can rapidly cross-link RBPs to RNA in HeLa cells, making it possible to also perform high-resolution timeresolved analyses in adherent cells.

\section{Protocol}

\section{TN150}

$50 \mathrm{mM}$ Tris $\mathrm{pH} 7.8$

$150 \mathrm{mM} \mathrm{NaCl}$

$0.1 \% \mathrm{NP}-40$

1X protease inhibitor

TN1000

$50 \mathrm{mM}$ Tris $\mathrm{pH} 7.8$

$1 \mathrm{M} \mathrm{NaCl}$

$0.1 \% \mathrm{NP}-40$

NP-PNK

50 mM Tris- $\mathrm{HCl}$ pH 7.8

$10 \mathrm{mM} \mathrm{MgCl} 2$

$0.1 \% \mathrm{NP}-40$

$5 \mathrm{mM}$ beta-mercaptoethanol

$5 \times$ PNK

250 mM Tris- $\mathrm{HCl}$ pH 7.8

$50 \mathrm{mM} \mathrm{MgCl} 2$

$50 \mathrm{mM}$ beta-mercaptoethanol

\section{WB I}

\section{0 mM Tris- $\mathrm{HCl} \mathrm{pH} 7.8$}

$300 \mathrm{mM} \mathrm{NaCl}$

$10 \mathrm{mM}$ imidazole

$6 \mathrm{M}$ guanidine- $\mathrm{HCl}$

$0.1 \% \mathrm{NP}-40$ 
$5 \mathrm{mM}$ beta-mercaptoethanol

WB II

50 mM Tris- $\mathrm{HCl}$ pH 7.8

$50 \mathrm{mM} \mathrm{NaCl}$

$10 \mathrm{mM}$ imidazole

$0.1 \% \mathrm{NP}-40$

$5 \mathrm{mM}$ beta-mercaptoethanol

\section{Elution buffer}

$50 \mathrm{mM}$ Tris $\mathrm{pH} 7.8$

$50 \mathrm{mM} \mathrm{NaCl}$

$250 \mathrm{mM}$ imidazole

$0.1 \%$ NP-40

$5 \mathrm{mM}$ beta-mercaptoethanol

\section{Protease K buffer}

$50 \mathrm{mM}$ Tris

$0.1 \% \mathrm{NP}-40$

$5 \mathrm{mM} \beta$-mercaptoethanol

$1 \%$ SDS

5 mM EDTA

$50 \mathrm{mM} \mathrm{NaCl} 2$

\section{Mammalian lysis buffer}

$50 \mathrm{mM}$ Tris-HCl pH 8

$100 \mathrm{mM} \mathrm{NaCl}$

$0.5 \%$ v/v Triton $X-100$

$0.25 \%$ w/v Na-deoxycholate

$0.1 \% \mathrm{w} / \mathrm{v}$ SDS

5 mM EDTA 
1 mM DTT (added fresh)

$1 \mathrm{X}$ protease inhibitor

Table 1: The buffers required for XCRAC and their compositions.

\section{UV cross-linking and lysate production}

1. Microorganisms in solution

1. Inoculate $3.5 \mathrm{~L}$ of the desired medium with yeast from an overnight culture to a starting $\mathrm{OD}_{600}$ of 0.05 . Grow at $30^{\circ} \mathrm{C}$ with continuous shaking at $180 \mathrm{rpm}$.

2. During the growth, prepare other necessary materials.

1. Prepare a container of liquid nitrogen.

2. Prepare $3 \mathrm{~L}$ of stress-inducing medium and warm to $30^{\circ} \mathrm{C}$ in a water bath.

3. Set up the filter apparatus, turn on the crosslinker (Figure 2A) and label $50 \mathrm{~mL}$ conical tubes, one for each timepoint.

3. Once the cells reach your desired $\mathrm{OD}_{600}$, pour 500 $\mathrm{mL}$ of cells straight into the cross-linker and UV irradiate with $250 \mathrm{~mJ}$ of $254 \mathrm{~nm}$ UV. See Figure 2A and Figure 3A for details on using the crosslinker. NOTE: The UV irradiation energy must be carefully optimized for each protein of interest. See the Discussion for further details.

4. After cross-linking, filter the cells using one of the vacuum filtration devices (Figure 2B,C). Roll up the membrane with the filtered cells, place in the $t=0$ (time zero) $50 \mathrm{~mL}$ conical tube, and flash-freeze in liquid nitrogen.
5. Filter the remaining cells over six different filters. Resuspend the collected cells in the $3 \mathrm{~L}$ of previously warmed stress-inducing medium by dropping the membranes in the medium and mixing vigorously with a stripette for $50 \mathrm{~s}$. After the $50 \mathrm{~s}$, prepare for taking the $\mathrm{t}=1$ sample.

6. After $1 \mathrm{~min}$, crosslink $500 \mathrm{~mL}$ of cells and harvest through filtration as in steps 1.1.3-1.1.4. Repeat after 2, 4, 8, 14, and $20 \mathrm{~min}$, or different timepoints as needed.

7. Store the conical tubes containing the cells at -80 ${ }^{\circ} \mathrm{C}$. Set phosphate buffered saline (PBS) at $4{ }^{\circ} \mathrm{C}$ overnight.

8. The next day, take each conical tube containing a crosslinked sample and resuspend the cells in $25 \mathrm{~mL}$ of cold PBS by shaking vigorously.

9. Transfer the cell suspensions to new conical tubes and spin at $4,600 \times g, 5 \mathrm{~min}$ at $4{ }^{\circ} \mathrm{C}$.

10. Pour off the PBS, quickly spin again to collect residual PBS and then decant the remaining liquid with a pipette.

11. Calculate the weight of the pellet in the tube by comparing it to an empty tube.

12. Add two pellet volumes of ice-cold TN150, $60 \mu \mathrm{L}$ of DNase 1 , and $10 \mu \mathrm{L}$ of RNase inhibitor. Incubate on ice for $30 \mathrm{~min}$. 
1. For example, for $400 \mathrm{mg}$ of cells, add $800 \mu \mathrm{L}$ of ice-cold TN150.

2. The addition of the DNase is not essential for most soluble proteins but is very important when studying chromatin-bound proteins such as RNA polymerase. Additionally, it reduces the viscosity of bacterial lysates. It is very important to use exactly two pellet volumes of the lysis buffer, or the lysis efficiency can decrease.

13. Add three pellet volumes (in $\mathrm{mL}$ ) of zirconia beads to the cell suspension. For yeast, use $0.5 \mathrm{~mm}$ diameter beads and for bacteria use $0.1 \mathrm{~mm}$.

1. For example, for $400 \mathrm{mg}$ of cells, measure out $1.2 \mathrm{~mL}$ of zirconia beads in a $1.5 \mathrm{~mL}$ tube and add them to the cells resuspended in lysis buffer.

14. Vortex the cell suspensions for $1 \mathrm{~min}$, then place on ice for $1 \mathrm{~min}$. Repeat for a total of $5 \mathrm{x}$.

15. Add two pellet volumes of TN150 buffer and vortex vigorously to mix.

16. Centrifuge the suspension in the conical tube at $4,600 \mathrm{~g}$ for $20 \mathrm{~min}$ at $4{ }^{\circ} \mathrm{C}$ in a benchtop centrifuge.

1. After centrifuging, take a $50 \mu \mathrm{L}$ sample of the supernatant for future Western blot analysis to examine the whole cell protein expression.

17. Transfer the supernatants into $1.5 \mathrm{~mL}$ tubes and spin the lysate for $20 \mathrm{~min}$ at $20,000 \times \mathrm{g}$ at $4{ }^{\circ} \mathrm{C}$, in a microfuge.

1. Alternatively, if using $5 \mathrm{~mL}$ tubes, centrifuge at $13,000 \times g$ for $20 \mathrm{~min}$.

2. Following centrifugation, take a $50 \mu \mathrm{L}$ sample of the supernatant for future Western blot analysis to examine the soluble expression of the protein.

18. Proceed to RBP capture (section 2).

2. Cultured adherent cells

1. Seed enough adherent cells in a Petri dish $24 \mathrm{~h}$ prior to UV cross-linking so that they can reach $80 \%$ confluency the next day. Grow overnight in the desired medium in a cell culture incubator at $37^{\circ} \mathrm{C}$, $5 \% \mathrm{CO}_{2}$.

NOTE: If using quartz Petri dishes, it is beneficial to promote cell adhesion through the treatment of the cultureware with poly-D-lysine $(70,000-140,000$ wt) and fetal calf serum (FCS) $2.5 \mathrm{~h}$ before seeding. Add enough poly-D-lysine to cover the entire growth surface and incubate at room temperature (RT) for 5 min. Next, the quartz Petri dish should be rinsed thoroughly with water and dried in the cell culture incubator for $2 \mathrm{~h}$ or until completely dry. Afterwards, add enough FCS to completely cover the growth surface and place in the incubator for at least 30 $\min$. The FCS should be completely removed before seeding cells.

2. Once the cells have reach $80 \%$ confluency, remove the media and wash with $15 \mathrm{~mL}$ of ice-cold PBS. Next, completely remove all remaining liquid and proceed immediately to the next step.

3. Transfer the Petri dish to the tray for adherent cells (Figure 3B) and UV irradiate with $300 \mathrm{~mJ}$ of 254 nm UV. See Figure 2A and Figure 3B for details on using the crosslinker.

NOTE: The UV irradiation energy must be carefully optimized for each protein of interest. See the Discussion for further details. 
4. Immediately after cross-linking, place the Petri dish on ice and add $10 \mathrm{~mL}$ of ice-cold PBS. Collect cells by scraping and transfer to a $15 \mathrm{~mL}$ conical tube. Pellet through centrifugation at $300 \times g$ for $5 \mathrm{~min}$ at $4{ }^{\circ} \mathrm{C}$.

5. Remove the PBS and resuspend the cell pellet in $1 \mathrm{~mL}$ of ice-cold PBS, and transfer to a 1.5 $\mathrm{mL}$ microcentrifuge tube. Pellet cells again by centrifugation for $5 \mathrm{~min}$ at $300 \times g$ at $4{ }^{\circ} \mathrm{C}$.

6. Remove the PBS and snap-freeze the cell pellets on dry ice. Store the cell pellets at $-80^{\circ} \mathrm{C}$ until required.

7. Repeat steps 1.2.3-1.2.6 for each timepoint.

8. Resuspend the cell pellets in $1 \mathrm{~mL}$ of lysis buffer and transfer to a $15 \mathrm{~mL}$ conical tube. Afterwards, add 1 $\mathrm{mL}$ of lysis buffer for a total of $2 \mathrm{~mL}$.

9. Add $5 \mu \mathrm{L}$ of mammalian RNase inhibitor.

10. Sonicate $5 x$ for $10 \mathrm{~s}$ on ice at $10 \mathrm{amp}$. Wait $30 \mathrm{~s}$ between sonication rounds.

11. Calculate the protein concentration of each sample and normalize to the lowest concentration.

12. Transfer $1.98 \mathrm{~mL}$ of lysate to a $2 \mathrm{~mL}$ tube.

13. Add $10 \mu \mathrm{L}$ of DNase I and incubate at $37^{\circ} \mathrm{C}$ for 5 min with shaking at 1,200 rpm.

14. Centrifuge the lysate at $16,000 \times g$ for $20 \mathrm{~min}$ at $4{ }^{\circ} \mathrm{C}$.

1. After centrifugation, take a $50 \mu \mathrm{L}$ sample of the supernatant for future Western blot analysis to examine soluble expression of the protein.

15. Proceed to RBP capture (section 2).

\section{RBP capture}

1. Wash the magnetic anti-FLAG $(75 \mu \mathrm{L}$ of slurry per sample) or IgG agarose (500 $\mu \mathrm{L}$ of slurry per sample) beads $3 \mathrm{x}$ with $5 \mathrm{~mL}$ of TN150. Resuspend in a final volume of $700 \mu \mathrm{L}$ of TN150 and add $100 \mu \mathrm{L}$ of washed beads to seven $15 \mathrm{~mL}$ conical tubes.

1. Store on ice until required.

2. Once the lysates have been clarified, add the supernatant to the tube containing the anti-FLAG/lgG beads.

3. Nutate at $4{ }^{\circ} \mathrm{C}$ for $2 \mathrm{~h}$.

NOTE: Some protocols describe overnight incubations with the beads, but this is not recommended, because long incubation times can dramatically reduce the recovery of cross-linked RNAs.

\section{Washing the beads and TEV cleavage of the tags}

1. Harvest the beads and remove the lysate.

1. Take a $50 \mu \mathrm{L}$ of sample of the supernatant for future Western blot analysis to examine the uncaptured protein.

2. Resuspend the beads in ice-cold TN1000 and transfer to a $1.5 \mathrm{~mL}$ tube. Wash for $10 \mathrm{~min}, 4^{\circ} \mathrm{C}$, with nutation. Repeat for a total of three washes.

1. If using IgG agarose beads, wash with $5 \mathrm{~mL}$ of TN1000. If using anti-FLAG beads, use $2 \mathrm{~mL}$.

3. Next, wash the beads $3 x$ with TN150, with the same volume as above. 
4. After the third wash, resuspend the beads in $600 \mu \mathrm{L}$ of TN150.

5. Add $30 \mathrm{U}$ of homemade GST-TEV protease to the bead suspension and rotate for $2 \mathrm{~h}$ at $\mathrm{RT}$.

NOTE: Recombinant GST-TEV protease is now also commercially available, but it has not been tested with this protocol.

1. During the digestion, prepare for the next steps by setting up columns of three $1.5 \mathrm{~mL}$ tubes for each sample (i.e. for seven samples, have three rows of seven columns).

2. To the final row of tubes, add $0.4 \mathrm{~g}$ of guanidium hydrochloride, $27 \mu \mathrm{L}$ of $5 \mathrm{M}$ sodium chloride and 3 $\mu \mathrm{L}$ of $2.5 \mathrm{M}$ imidazole $(\mathrm{pH}=8)$. Note that the $\mathrm{pH}$ of the imidazole must be 8 . This is critical to maintain RNA integrity.

3. Additionally, wash the required volume of nickel beads in WB I 3x. Use $100 \mu \mathrm{L}$ of slurry per sample. After the final wash, resuspend the beads in the same original volume of WB I and store on ice.

6. Once the TEV digestion is complete, collect the supernatant using a magnetic rack for anti-FLAG beads or centrifugation for IgG beads, and transfer to the first row of the tubes previously set up.

1. Take a $50 \mu \mathrm{L}$ of sample of the TEV eluate for Western blot analysis.

7. Set a thermoblock incubator to $37^{\circ} \mathrm{C}$. To the second row of tubes, add $1 \mu \mathrm{L}$ of RNase cocktail (1:50 dilution).

8. Take $550 \mu \mathrm{L}$ of TEV eluate from the first row of tubes and add to the second row (containing the RNase cocktail). Pipette vigorously to ensure mixing.
9. After completing this for the first sample, immediately place the tube into the thermoblock and start a timer. Move on to the subsequent samples, such that each is staggered.

10. Incubate for exactly 5 min. Once completed, remove the first sample from the thermoblock and transfer the solution to the third row of tubes (containing the guanidium hydrochloride powder).

NOTE: A 5 min incubation with a 1:50 dilution of the RNase cocktail is usually suitable for most proteins, but this step will need to be carefully optimized with different incubation times or concentrations for each protein to make sure that the cross-linked RNAs are of the correct size (30-100 nt).

11. Immediately vortex for a couple of seconds at full speed to dissolve the guanidium powder and then move on to the next sample.

12. After all samples have been transferred into the guanidium powder, vortex again to ensure all the powder is fully dissolved.

13. Add $100 \mu \mathrm{L}$ of washed nickel beads and rotate overnight at $4{ }^{\circ} \mathrm{C}$. This incubation can be shortened to $2 \mathrm{~h}$.

\section{On-bead alkaline phosphatase treatment}

1. Set a thermoblock to $37^{\circ} \mathrm{C}$.

2. Place a purification spin column in a $2 \mathrm{~mL}$ tube, one for each sample. Transfer the nickel beads to the columns and allow the supernatant to drain through. Afterwards, ensure that all the nickel beads were removed from the $1.5 \mathrm{~mL}$ tube by rinsing with WB I and applying to the column. 
3. Set up $2 \mathrm{~mL}$ tubes, six per sample (one to collect each wash). Keep the outside of the columns dry to maintain flow. Wash the beads $3 x$ with $500 \mu \mathrm{L}$ of WB I and then $3 x$ with $500 \mu \mathrm{L}$ of NP-PNK.
4. Close the lid of the spin column and briefly spin beads to remove excess buffer.

5. Put the stopper on the column, put columns into $1.5 \mathrm{~mL}$ tubes and add $60 \mu \mathrm{L}$ of the reaction mixture seen in Table 2.

\begin{tabular}{|c|c|c|}
\hline Component & $\mathbf{1 x}$ & $\mathbf{7 . 5 x}$ \\
\hline 5 x PNK buffer & 12 & 30 \\
\hline Alkaline phosphatase & 4 & 15 \\
\hline RNase inhibitor & 2 & 315 \\
\hline $\mathrm{H}_{2} \mathrm{O}$ & 42 & $\mathbf{4 5 0} \boldsymbol{\mu L}$ \\
\hline
\end{tabular}

Table 2: Alkaline phosphatase reaction mixture.

6. Incubate the beads for $1 \mathrm{~h}$ at $37^{\circ} \mathrm{C}$.

7. Wash the beads $1 \mathrm{x}$ with $500 \mu \mathrm{L}$ of $\mathrm{WB}$ I to inactivate the alkaline phosphatase and then $3 x$ with $500 \mu \mathrm{L}$ of NP-PNK buffer. Make sure to thoroughly rinse the inside of the column with the NP-PNK buffer to remove any traces of guanidium.

\section{On-bead ligation of the App-PE linker to the 3' end of the RNA}

1. Spin out the remaining buffer and add $60 \mu \mathrm{L}$ of the mixture specified in Table 3 (see Table 4 for the App-PE sequence) to the columns. Incubate the reaction for $6 \mathrm{~h}$ at $25^{\circ} \mathrm{C}$.

\begin{tabular}{|c|c|c|}
\hline Component & $\mathbf{1 x}$ & $\mathbf{7 . 5 x}$ \\
\hline 5 x PNK buffer & 12 & 40 \\
\hline App-PE adapter $(100 \mu \mathrm{M})$ & 0.6 & 22.5 \\
\hline T4 RNA ligase 2 truncated K227Q & 3 & 11.25 \\
\hline RNase inhibitor & 1.5 & 90 \\
\hline $50 \%$ PEG 8000 & 12 & 231.75 \\
\hline
\end{tabular}


Table 3: App-PE linker ligation reaction mixture.

\begin{tabular}{|c|c|}
\hline Oligonucleotide name & Sequence (5'-3') \\
\hline L5Aa & invddT-ACACrGrArCrGrCrUrCrUrUrCrCrGrArUrCrUrNrNrNrUrArArGrCrN-OH \\
\hline $\mathrm{L} 5 \mathrm{Ab}$ & invddT-ACACrGrArCrGrCrUrCrUrUrCrCrGrArUrCrUrNrNrNrArUrUrArGrCrN-OH \\
\hline L5Ac & invddT-ACACrGrArCrGrCrUrCrUrUrCrCrGrArUrCrUrNrNrNrGrCrGrCrArGrCrN-OH \\
\hline L5Ad & invddT-ACACrGrArCrGrCrUrCrUrUrCrCrGrArUrCrUrNrNrNrCrGrCrUrUrArGrCrN-OH \\
\hline L5Ba & invddT-ACACrGrArCrGrCrUrCrUrUrCrCrGrArUrCrUrNrNrNrArGrArGrCrN-OH \\
\hline $\mathrm{L} 5 \mathrm{Bb}$ & invddT-ACACrGrArCrGrCrUrCrUrUrCrCrGrArUrCrUrNrNrNrGrUrGrArGrCrN-OH \\
\hline L5Bc & invddT-ACACrGrArCrGrCrUrCrUrUrCrCrGrArUrCrUrNrNrNrCrArCrUrArGrCrN-OH \\
\hline L5Bd & invddT-ACACrGrArCrGrCrUrCrUrUrCrCrGrArUrCrUrNrNrNrUrCrUrCrUrArGrCrN-OH \\
\hline L5Ca & invddT-ACACrGrArCrGrCrUrCrUrUrCrCrGrArUrCrUrNrNrNrCrUrArGrCrN-OH \\
\hline $\mathrm{L} 5 \mathrm{Cb}$ & invddT-ACACrGrArCrGrCrUrCrUrUrCrCrGrArUrCrUrNrNrNrUrGrGrArGrCrN-OH \\
\hline L5Cc & invddT-ACACrGrArCrGrCrUrCrUrUrCrCrGrArUrCrUrNrNrNrArCrUrCrArGrCrN-OH \\
\hline L5Cd & invddT-ACACrGrArCrGrCrUrCrUrUrCrCrGrArUrCrUrNrNrNrGrArCrUrUrArGrCrN-OH \\
\hline L5Da & invddT-ACACrGrArCrGrCrUrCrUrUrCrCrGrArUrCrUrNrNrNrCrGrUrGrArUrN-OH \\
\hline $\mathrm{L} 5 \mathrm{Db}$ & invddT-ACACrGrArCrGrCrUrCrUrUrCrCrGrArUrCrUrNrNrNrGrCrArCrUrArN-OH \\
\hline L5Dc & invddT-ACACrGrArCrGrCrUrCrUrUrCrCrGrArUrCrUrNrNrNrUrArGrUrGrCrN-OH \\
\hline L5Dd & invddT-ACACrGrArCrGrCrUrCrUrUrCrCrGrArUrCrUrNrNrNrArUrCrArCrGrN-OH \\
\hline L5Ea & invddT-ACACrGrArCrGrCrUrCrUrUrCrCrGrArUrCrUrNrNrNrCrArCrUrGrUrN-OH \\
\hline L5Eb & invddT-ACACrGrArCrGrCrUrCrUrUrCrCrGrArUrCrUrNrNrNrGrUrGrArCrArN-OH \\
\hline L5Ec & invddT-ACACrGrArCrGrCrUrCrUrUrCrCrGrArUrCrUrNrNrNrUrGrUrCrArCrN-OH \\
\hline L5Ed & invddT-ACACrGrArCrGrCrUrCrUrUrCrCrGrArUrCrUrNrNrNrArCrArGrUrGrN-OH \\
\hline App_PE & App-NAGATCGGAAGAGCACACGTCTG-ddC \\
\hline
\end{tabular}

Table 4: The sequences of the DNA and RNA adaptors required for ligation onto the 5' and 3' ends of captured RNAs. These were purified through RNase-free HPLC. 
2. Wash beads $1 \mathrm{x}$ with $500 \mu \mathrm{L}$ of $\mathrm{WB} I$ and $3 \mathrm{x}$ with $500 \mu \mathrm{L}$ of NP-PNK buffer. Put the column in a new tube and spin out the remaining buffer.

\section{On-bead phosphorylation of the 5 ' ends of the RNA}

1. Add $80 \mu \mathrm{L}$ of the mixture specified in Table $\mathbf{5}$ to the columns. Incubate the reaction for $40 \mathrm{~min}$ at $37^{\circ} \mathrm{C}$.
NOTE: The samples will now be highly radioactive.

Thus, all subsequent work should be performed behind a protective screen and waste should be disposed of according to local health and safety rules.

\begin{tabular}{|c|c|c|}
\hline Component & $1 x$ & $7.5 x$ \\
\hline $5 \times$ PNK buffer & 16 & 120 \\
\hline${ }^{32}$ P-үATP $(10 \mu \mathrm{Ci} / \mu \mathrm{L})$ & 3 & 22.5 \\
\hline T4 PNK & 3 & 22.5 \\
\hline $\mathrm{H}_{2} \mathrm{O}$ & 58 & 435 \\
\hline Final volume & $80 \mu L$ & $600 \mu \mathrm{L}$ \\
\hline
\end{tabular}

Table 5: Phosphorylation reaction mixture.

2. Add $1 \mu \mathrm{L}$ of $100 \mathrm{mM}$ ATP and let the reaction proceed for another $20 \mathrm{~min}$. This will make sure that almost all of the $5^{\prime}$ ends have phosphates to facilitate ligation of the 5 ' linker.

3. Set up $2 \mathrm{~mL}$ tubes, five per sample.

4. Wash beads $1 \mathrm{x}$ with $500 \mu \mathrm{L}$ of WB I and $3 \mathrm{x}$ with 500 $\mu \mathrm{L}$ of NP-PNK buffer. Note that these elutions will be very radioactive and therefore should be disposed of appropriately.

5. Move the column to the final tube and spin out the remaining buffer.

\section{On-bead ligation of the 5' linker}

NOTE: The ${ }^{\prime}$ linkers contain an RNA barcode that is used for identification of each sample after sequencing. Thus, it is absolutely crucial to note which linker is used for which sample.

1. Add $78 \mu \mathrm{L}$ of the mixture described in Table 6 to the columns. Add $2 \mu \mathrm{L}$ of 5 ' adapter (100 $\mu \mathrm{M}$; see Table 4) to each tube and incubate overnight at $18^{\circ} \mathrm{C}$.

\begin{tabular}{|c|c|c|}
\hline Component & $\mathbf{1 x}$ & $\mathbf{7 . 5 x}$ \\
\hline $5 \times$ PNK buffer & 16 & 120 \\
\hline
\end{tabular}




\begin{tabular}{|c|c|c|}
\hline ATP $(10 \mathrm{mM})$ & 8 & 60 \\
\hline RNase inhibitor & 2 & 15 \\
\hline T4 RNA ligase & 4 & 30 \\
\hline $\mathrm{H}_{2} \mathrm{O}$ & 48 & 360 \\
\hline Final volume & $\mathbf{7 8} \boldsymbol{\mu L}$ & $\mathbf{5 8 5} \boldsymbol{\mu L}$ \\
\hline
\end{tabular}

Table 6: 5 ' linker ligation reaction mixture.

2. The following day, wash beads $1 \mathrm{x}$ with $500 \mu \mathrm{L}$ of WB I and $3 x$ with $500 \mu \mathrm{L}$ of WB II and transfer the columns to a new $2 \mathrm{~mL}$ tube.

\section{Elution, SDS-PAGE, and RNA extraction}

1. Set the centrifuge to $4{ }^{\circ} \mathrm{C}$. Prepare two rows of $1.5 \mathrm{~mL}$ tubes per sample for elution.

2. Spin out the void volume of the columns with nickel beads with a quick spin. Place the columns in the first row of elution tubes and add $200 \mu \mathrm{L}$ of elution buffer. Wait $2 \mathrm{~min}$, then force the buffer through the column with a quick spin.

3. Move the columns to the second row of tubes and repeat step 8.2. Each sample will now have $400 \mu \mathrm{L}$ of eluate in total, split over two $1.5 \mathrm{~mL}$ tubes.

1. Take all the eluates and transfer them together to a $5 \mathrm{~mL}$ tube. Add $2 \mu \mathrm{L}$ of $20 \mathrm{mg} / \mathrm{mL}$ of glycogen. Thus, if using seven samples, there will now be $2.8 \mathrm{~mL}$ of pooled eluate in the $5 \mathrm{~mL}$ tube.

4. Add $100 \mu \mathrm{L}$ of trichloroacetic acid (TCA) per sample [e.g. $700 \mu \mathrm{L}$ of TCA for 7 samples $(2.8 \mathrm{~mL}$ of pooled eluate)] to the $5 \mathrm{~mL}$ tube, and vortex well for $30 \mathrm{~s}$.

5. Incubate on ice for $20 \mathrm{~min}$.
6. Centrifuge for 30 min at $17,000 \times g, 4^{\circ} \mathrm{C}$, in a benchtop centrifuge.

7. Carefully remove the supernatant from the conical tube, checking the pipette with a Geiger counter to ensure the pellet has not been removed accidentally. If it has, return the supernatant to the tube and centrifuge for another 10 $\min$.

NOTE: The supernatant might still be highly radioactive. Make sure to use proper shielding.

8. Fully resuspend the pellet in $2 \mathrm{~mL}$ of ice-cold acetone.

9. Centrifuge for $15 \mathrm{~min}$ at $17,000 \times \mathrm{g}, 4^{\circ} \mathrm{C}$.

10. Remove as much of the acetone with a $\mathrm{P} 1000$ pipette as possible. Afterwards, briefly spin the tube to gather small droplets of acetone, and then remove with a P10 pipette. Dry for 2 min in a fume hood.

NOTE: The acetone supernatant can still be radioactive. Make sure to use proper shielding.

11. Resuspend the sample in $30 \mu \mathrm{L}$ of $1 \mathrm{x}$ protein loading buffer. To ensure that the pellet is properly resuspended, check that the vast majority of the radioactivity is now present in the loading buffer and not left in the $1.5 \mathrm{~mL}$ tube by removing the solution in a P200 pipette and measuring the activity left in the $1.5 \mathrm{~mL}$ tube using a Geiger counter. 
12. Heat the sample for $10 \mathrm{~min}$ at $65^{\circ} \mathrm{C}$. Load on a $1 \mathrm{~mm}$, $4-12 \%$ precast Bis-Tris gel and run for $1.5 \mathrm{~h}$ at $125 \mathrm{~V}$ in MOPS buffer.

13. After the gel has finished running, open the gel cassette. The gel should be retained on the bottom plate. Dispose of the top.

14. Wrap the gel in cling film and then secure it using tape to the inside of a light-tight cassette. Ensure that the cassette has an amplifying screen to improve the signal.

15. Expose an autoradiographic film to the gel and store the cassette at $-80^{\circ} \mathrm{C}$ during the exposure. The exposure time will vary between proteins with different cross-linking efficiencies.

1. When placing the film, there must be a way of realigning it to the cassette in order to cut out the band of interest in the subsequent step. To ensure this, use a fluorescent ruler and also ensure that the gel is at a corner of the cassette, which is then covered by the film also placed in the utmost corner. NOTE: As a rule of thumb, eluates in loading buffer that give a reading of at least $\sim 250 \mathrm{cps}$ when displayed to a Geiger counter give sufficient signal for an exposure of $3 \mathrm{~h}$. Otherwise, overnight exposure is performed.

16. Develop the film. Cut away the cling film covering the gel but do not move the gel. Otherwise, the image will be offset from the gel.

NOTE: The gel will likely be highly radioactive. Make sure to use proper shielding when cutting out the gel slice.

17. Place the film over the gel and excise the band of interest. Put the gel slice into a $2 \mathrm{~mL}$ tube.
18. Smash the gel slice using a P1000 pipette tip and add $600 \mu \mathrm{L}$ of proteinase $\mathrm{K}$ buffer plus $200 \mu \mathrm{g}$ of proteinase $\mathrm{K}$ (this protocol uses $10 \mu \mathrm{L}$ of a $20 \mathrm{mg} / \mathrm{mL}$ proteinase $\mathrm{K}$ solution). Incubate for $2 \mathrm{~h}$ at $55^{\circ} \mathrm{C}$ with vigorous shaking.

19. Afterwards, cut the end of a P1000 tip off with a clean scalpel and transfer the supernatant and gel pieces to a spin column placed in a $2 \mathrm{~mL}$ tube.

20. Spin the column for $1 \mathrm{~min}$ at $17,000 \times g$ at RT. Collect the flow-through, which contains the radioactive, isolated RNAs.

21. Perform a phenol:chloroform extraction.

1. Add $50 \mu \mathrm{L}$ of $3 \mathrm{M}$ sodium acetate, $\mathrm{pH}=5.2$, and 500 $\mu \mathrm{L}$ of phenol:chloroform and vortex well. Spin for 5 $\min$ at $17,000 \times g$. Remove the aqueous top layer and place in a new $1.5 \mathrm{~mL}$ tube.

2. Add $500 \mu \mathrm{L}$ of chloroform and vortex vigorously for $10-15 \mathrm{~s}$. Spin for $5 \mathrm{~min}$ at $17,000 \mathrm{x} g$ at RT. Remove the aqueous layer and place in a new $1.5 \mathrm{~mL}$ tube.

3. Add $1 \mu \mathrm{L}$ of $20 \mathrm{mg} / \mathrm{mL}$ glycogen and $1 \mathrm{~mL}$ of icecold, $96 \%$ ethanol. Precipitate for $30 \mathrm{~min}$ at $-80{ }^{\circ} \mathrm{C}$ or overnight at $-20^{\circ} \mathrm{C}$.

4. Centrifuge for $30 \mathrm{~min}$ at $4{ }^{\circ} \mathrm{C}, 17,000 \times \mathrm{g}$. Remove the supernatant, add $500 \mu \mathrm{L}$ of $70 \%$ ethanol and centrifuge for $5 \mathrm{~min}, 4^{\circ} \mathrm{C}$ at $17,000 \times \mathrm{g}$. Remove all the ethanol, perform a quick spin to gather residue and remove excess with a $\mathrm{P} 10$ pipette.

5. Dry the pellet for $\sim 3$ min in a fume hood. Resuspend in $20 \mu \mathrm{L}$ of DEPC-treated water.

22. Store the RNA at $-80{ }^{\circ} \mathrm{C}$ overnight or proceed immediately to the reverse transcription step. 


\section{Reverse transcription}

1. Add $2 \mu \mathrm{L}$ of $10 \mu \mathrm{M} \mathrm{RT}$ oligo (PE_reverse; see Table 7) and $4 \mu \mathrm{L}$ of $5 \mathrm{mM}$ dNTPs to the $20 \mu \mathrm{L}$ of RNA.

\begin{tabular}{|c|c|}
\hline Oligonucleotide name & Sequence (5'-3') \\
\hline P5 forward & AATGATACGGCGACCACCGAGATCTACACTCTTTCCCTACACGACGCTCTTCCGATCT \\
\hline BC1 & CAAGCAGAAGACGGCATACGAGATCGTGATGTGACTGGAGTTCAGACGTGTGCTCTTCCGATC \\
\hline BC3 & CAAGCAGAAGACGGCATACGAGATGCCTAAGTGACTGGAGTTCAGACGTGTGCTCTTCCGATC \\
\hline BC4 & CAAGCAGAAGACGGCATACGAGATTGGTCAGTGACTGGAGTTCAGACGTGTGCTCTTCCGATC \\
\hline BC5 & CAAGCAGAAGACGGCATACGAGATCACTGTGTGACTGGAGTTCAGACGTGTGCTCTTCCGATC \\
\hline BC7 & CAAGCAGAAGACGGCATACGAGATCAGATCGTGACTGGAGTTCAGACGTGTGCTCTTCCGATC \\
\hline BC8 & CAAGCAGAAGACGGCATACGAGATTAGCTTGTGACTGGAGTTCAGACGTGTGCTCTTCCGATCT \\
\hline BC9 & CAAGCAGAAGACGGCATACGAGATGATCAGGTGACTGGAGTTCAGACGTGTGCTCTTCCGATC \\
\hline BC10 & CAAGCAGAAGACGGCATACGAGATATCACGGTGACTGGAGTTCAGACGTGTGCTCTTCCGATC† \\
\hline PE_reverse & CAGACGTGTGCTCTTCCGATCT \\
\hline
\end{tabular}

Table 7: The PCR primers (including the barcode sequences) and the reverse transcription primer.

2. Transfer to a preheated thermoblock at $85^{\circ} \mathrm{C}$ for $3 \mathrm{~min}$, then snap-chill on ice for $5 \mathrm{~min}$. Collect the contents of the tube by brief centrifugation and then add $8 \mu \mathrm{L}$ of $5 \mathrm{x}$ reverse transcriptase buffer, $2 \mu \mathrm{L}$ of $100 \mathrm{mM}$ DTT, and 2 $\mu \mathrm{L}$ of RNase inhibitor.

3. Incubate the mixture at $50{ }^{\circ} \mathrm{C}$ for 3 min and then add 2 $\mu \mathrm{L}$ of reverse transcriptase and incubate for $1 \mathrm{~h}$ at $50^{\circ} \mathrm{C}$.

4. Inactivate the reverse transcriptase by incubation at 65 ${ }^{\circ} \mathrm{C}$ for $15 \mathrm{~min}$.

5. Transfer the tubes to a preheated thermoblock at $37^{\circ} \mathrm{C}$ and leave for $3 \mathrm{~min}$ to acclimatize.

6. Add $2 \mu \mathrm{L}$ of RNase $\mathrm{H}$ and incubate for $30 \mathrm{~min}$ at $37^{\circ} \mathrm{C}$.
7. Isolate the cDNA using SPRI beads.

1. Add two volumes of $84 \mu \mathrm{L}$ of beads. Incubate for 15 min. Put the beads on a magnetic rack and leave for 1 min to harvest the beads.

2. Remove and dispose of the supernatant and add $200 \mu \mathrm{L}$ of $70 \%$ ethanol. Do not remove the beads from the magnetic rack. Incubate the beads with the ethanol for $30 \mathrm{~s}$.

3. Remove the ethanol and repeat the wash step. Remove all the residual ethanol using a P10 tip.

4. Put the beads in a fume hood for 2 min to dry them. Remove the beads from the rack, resuspend them 
in $12 \mu \mathrm{L}$ of water, and then put the beads back on the rack. Remove $11 \mu \mathrm{L}$ of supernatant.

8. Freeze the $\mathrm{CDNA}$ at $-20^{\circ} \mathrm{C}$ or proceed immediately to the PCR step.

\section{0. $q P C R$ reaction}

1. Prior to the final PCR for amplification of the cDNAs, a quantitative polymerase chain reaction ( $q P C R$ ) is performed to identify the optimal number of cycles for amplifying the cDNAs to prevent overamplification of the library.

2. Set up a qPCR reaction on ice according to Table 8. See Table 7 for all primers.

\begin{tabular}{|c|c|}
\hline Component & $1 \mathbf{x}$ \\
\hline $2 \times$ qPCR reaction mastermix & 5 \\
\hline $0.1 \mu \mathrm{M}$ P5 primer (forward) & 0.8 \\
\hline $0.1 \mu \mathrm{M} \mathrm{BC}$ primer (reverse) & 0.8 \\
\hline CDNA (or water as a negative control) & 1 \\
\hline $\mathrm{H}_{2} \mathrm{O}$ & 2.4 \\
\hline Final volume & $10 \mu \mathrm{L}$ \\
\hline
\end{tabular}

Table 8: qPCR reaction mixture.

3. For proper quantification of the cycles needed for amplification, use three technical replicates for the cDNA and three negative (i.e. water) controls.

4. Seal the plates with optically transparent film and run the qPCR according to the kit manufacturer's instructions.

5. Analyze the samples through an absolute quantification method to identify the number of cycles $(n)$ at which the knee of exponential growth is reached (see Figure 4C for an example). This number of cycles is then used for the final amplification of the rest of the cDNA.

\section{PCR reaction and gel extraction}

1. Set up the PCR reaction on ice according to Table 9. See Table 7 for all primers.

NOTE: Only $5 \mu \mathrm{L}$ of the cDNA library is used.

\begin{tabular}{|c|c|}
\hline Component & $\mathbf{1 x}$ \\
\hline $10 x$ proof-reading polymerase buffer & 5 \\
\hline $10 \mu \mathrm{M}$ P5 primer (forward) & 1 \\
\hline $10 \mu \mathrm{M} \mathrm{BC}$ primer (reverse) & 1 \\
\hline
\end{tabular}




\begin{tabular}{|c|c|}
\hline $5 \mathrm{mM}$ dNTPs & 2.5 \\
\hline Proof reading polymerase enzyme & 1 \\
\hline CDNA & 5 \\
\hline $\mathrm{H}_{2} \mathrm{O}$ & 34.5 \\
\hline Final volume & $\mathbf{5 0 \mu L}$ \\
\hline
\end{tabular}

Table 9: PCR reaction mixture.

2. Run PCR as follows: $95^{\circ} \mathrm{C}$ for 2 min; $\mathrm{n}$ cycles of $98^{\circ} \mathrm{C}$ for $20 \mathrm{~s}, 52^{\circ} \mathrm{C}$ for $30 \mathrm{~s}$, and $72{ }^{\circ} \mathrm{C}$ for $1 \mathrm{~min}$; and $72{ }^{\circ} \mathrm{C}$ for 5 $\min$. The number $(n)$ of cycles for amplifying the XCRAC library is determined by the qPCR described in section 10.

3. Add $1 \mu \mathrm{L}$ of exonuclease I and incubate at $37^{\circ} \mathrm{C}$ for 60 $\min$.

4. Clean up the amplified cDNA using SPRI beads as described above using two volumes of beads (i.e. 100 $\mu \mathrm{L})$. Elute in $11 \mu \mathrm{L}$.

5. Add $3 \mu \mathrm{L}$ of $6 \mathrm{x}$ loading dye and run on a precast $6 \%$ TBE gel at $100 \mathrm{~V}$ for $1 \mathrm{~h}$ in $1 \mathrm{x}$ TBE buffer. Use a ladder appropriate for quantification of short DNA fragments.

6. Once finished, remove the gel from the cassette and place in a suitable, liquid-tight container with enough $1 \mathrm{x}$ TBE to cover the gel (e.g. $\sim 50 \mathrm{~mL}$ ). Add an appropriate amount of SYBR safe dye (e.g. for $50 \mathrm{~mL}$, use $5 \mu \mathrm{L}$ of a $10,000 x$ dye)

7. Allow the gel to stain through gentle mixing for $15 \mathrm{~min}$ at RT. Drain the SYBR-containing 1x TBE and replace with clean 1x TBE. Wash the gel for 10 min with gentle shaking at RT.

8. Drain the $1 \mathrm{x}$ TBE and place the gel in a transparent folder. Cut the folder to an appropriate size.
9. Image the gel through an appropriate means such as a phosphorimager. Excise DNA fragments between 175 bp and $\sim 400 \mathrm{bp}$. Put the gel slice in a $1.5 \mathrm{~mL}$ tube.

10. Thoroughly smash the gel slice using a P1000 tip and add $400 \mu \mathrm{L}$ of $\mathrm{H}_{2} \mathrm{O}$. Incubate at $37^{\circ} \mathrm{C}$ with shaking for 1 $\mathrm{h}$ in a thermoblock.

11. Freeze the sample on dry ice for $10 \mathrm{~min}$, then place back in the thermoblock at $37^{\circ} \mathrm{C}$ with shaking for $1 \mathrm{~h}$.

12. Create a filter unit by taking a filter column and inserting two glass microfiber filters inside. Place the unit in a 1.5 $\mathrm{mL}$ tube.

13. Cut off the end of a P1000 tip with a clean scalpel and uptake the smashed TBE gel suspension, then dispense into the filter unit created in step 11.12. Spin at 17,000 $x g$ for $30 \mathrm{~s}$.

14. Add $1 \mu \mathrm{L}$ of glycogen to the supernatant, along with 40 $\mu \mathrm{L}$ of sodium acetate, $\mathrm{pH}=5.2$, and $1 \mathrm{~mL}$ of $96 \%$ ethanol. Incubate at $-80{ }^{\circ} \mathrm{C}$ for $30 \mathrm{~min}$.

15. Centrifuge for $30 \mathrm{~min}$ at $17,000 \times g, 4{ }^{\circ} \mathrm{C}$. Discard the supernatant and wash with $500 \mu \mathrm{L}$ of $70 \%$ ethanol.

16. Spin for $5 \mathrm{~min}$, remove the ethanol entirely and then dry the pellet in a fume hood for $3 \mathrm{~min}$. 
17. Resuspend in $10 \mu \mathrm{L}$ of $\mathrm{H}_{2} \mathrm{O}$ and measure the DNA concentration.

\section{Representative Results}

To demonstrate the efficacy of the XCRAC method, a timecourse experiment with yeast strains expressing an HTPtagged Nrd1 protein was performed. A detailed schematic representation describing how the method works is provided in Figure 1. Like Nab3, Nrd1 is involved in nuclear RNA decay of a variety of RNA transcripts ${ }^{37}$. Previous work from the Corden lab suggested that Nrd1 binding to its RNA targets changes significantly when cells are subjected to glucose starvation ${ }^{28,38}$. As such, cells growing exponentially in medium containing glucose (SD-TRP) were shifted to the same medium without glucose (S-TRP) over a time-course to monitor dynamic changes in Nrd1-RNA interactions. Samples were taken and cross-linked in the Vari-X-linker chamber (Figure 3A) before the shift and then after 1, 2, 4, 8, 14, and $20 \mathrm{~min}$. The medium used for cell growth was deliberately deficient in tryptophan to reduce UV absorption by this aromatic amino acid. Note that it is best to use synthetic medium that is filter sterilized as because autoclaving the medium can lead to caramelization of the sugars. This then reduces the cross-linking efficiency.

Figure 4A shows a representative autoradiograph from a XCRAC experiment. Note that in this example, the samples were not pooled together. Instead, each was run individually on the gel. This is recommended for initial experimental tests to show that the protein cross-links effectively to RNA at all of the tested timepoints. A particularly intense signal was observed at the expected molecular weight of the RBP, representing the protein bound to very short, radiolabeled RNAs not amenable for sequencing. Therefore, the smeary signal above this band, which is the protein crosslinked to longer RNA fragments, was isolated. The fragment was cut from just above the protein band plus around $30 \mathrm{kDa}$. Figure 4B shows an autoradiogram following excision, with the protein cross-linked to short RNAs left in the gel and the previously smeary signal now excised.

After reverse transcription, the cDNA library must be amplified using PCR. However, overamplification of the library must be avoided as this can introduce bias towards sequences preferentially amplified by the polymerase and generate PCR artefacts. Overamplified libraries also contain a large number of duplicate sequences that waste reads on the sequencer. In order to calculate the ideal number of PCR cycles for amplification of the final library, an aliquot of the cDNA was amplified through $\mathrm{gPCR}$ using the P5 and BC oligonucleotides. The first cycle at which the library reached peak fluorescence was chosen as the PCR cycle count. Figure 4C gives an example of a $\mathrm{qPCR}$ from a typical cDNA library, which yielded a peak cycle count of 16 . This value was then used for the final XCRAC PCR. In order to process the sequenced data, we used software previously developed in our lab (pyCRAC) and the corresponding pipeline for analysis of the kinetic CRAC data (Nues et al., 2017; https://git.ecdf.ed.ac.uk/sgrannem/ pycrac, https://bitbucket.org/sgrann/kinetic_crac_pipeline/ src/default/). These open source software tools enable demultiplexing and trimming of the data, removal of PCR duplicates, identification of statistically significant peaks, cluster reads into contiguous sequences, and identify binding motifs $^{39}$. Further details of how these tools operate are found on their respective webpages.

We also started to develop a XCRAC protocol for mammalian cells. The majority of mammalian cell lines are grown as a monolayer and the tray in our crosslinker with the UV- 
permeable bag is not suitable for experiments with adherent cells. To overcome this problem, we developed a stage where users can UV irradiate 1-2 Petri dishes (150 mm diameter and $25 \mathrm{~mm}$ in depth) with adherent cells (Figure 3B). As a first test, the efficiency of the cross-linker for mammalian cells was measured through cross-linking and capture of stably tagged GFP-RBM7 using anti-GFP antibodies and a traditional CLIP-based purification. As shown in Figure 5A, the cross-linker was able to recover protein-RNA complexes from mammalian cells grown as a monolayer using 254 $\mathrm{nm}$ UV irradiation at efficiencies comparable to a widely used UV irradiation device. However, standard cell culture plasticware normally used for UV cross-linking experiments is impenetrable to $254 \mathrm{~nm}$ UV. Therefore, in our crosslinker the cells would only receive irradiation from the upper bank of UV lamps. To overcome this, we developed a UVpermeable quartz Petri dish for cell growth and cross-linking. Use of the quartz cultureware displayed robust recovery of protein-RNA complexes with as few as $2 \mathrm{~s}$ of UV irradiation (Figure 5B). When combined with RBP capture methods for mammalian cells such as CLIP technologies, these short cross-linking times are amenable with timecourses to recover spatiotemporal RNA-binding profiles of RBPs in response to genotoxic stresses or rapid depletions of protein factors, or in parallel with transcriptional or cell cycle synchronization.

Figure 6 shows several examples of the Nrd1 data processed by the XCRAC pipeline. This figure was prepared using the bedgraph files generated by the pipeline and the python GenomeBrowser package (https://pypi.org/project/ GenomeBrowser/1.6.3/), which we designed to simplify making publication-quality genome browser images of the data. The grey rectangles represent genomic regions that expressed noncoding RNAs, such as the cryptic unstable transcript (CUTs), stable uncharacterized transcripts $(\mathrm{SUTs})^{40}$, and Xrn1-sensitive unstable transcripts (XUTs) ${ }^{41}$. The data in Figure 6 show that Nrd1 binds to many of these noncoding RNA transcripts, consistent with the idea that this protein is involved in degradation of this class of transcripts 42 . Figure $6 \mathrm{~A}$ shows a $\sim 15 \mathrm{~kb}$ region on chromosome IV. Here there was a significant increase in binding of $\mathrm{Nrd1}$ to transcripts encoding the high-affinity glucose transporters HXT6 and HXT7, both of which are upregulated during glucose starvation. It is likely that transcription termination by the NNS complex may influence the induction kinetics of these genes during glucose starvation. Figure 6B shows an example of Nrd1 cross-linking to the Imd3 transcript, which is known to be regulated by $\mathrm{Nab}^{43}$. In this case the data demonstrated a significant reduction in binding upon glucose starvation. Previous work showed decreased binding of Nab3 to the Tye 7 transcript during glucose starvation ${ }^{44}$. Consistent with this observation, the $\mathrm{XCRAC}$ data suggest that binding of Nrd1 decreased during glucose starvation and Nrd1 crosslinking to Tye7 was at its lowest after 8 min of stress (Figure 4C). However, it appears that this effect was only transient, because after 14 min of glucose starvation, Nrd1 binding went back to starting levels. 


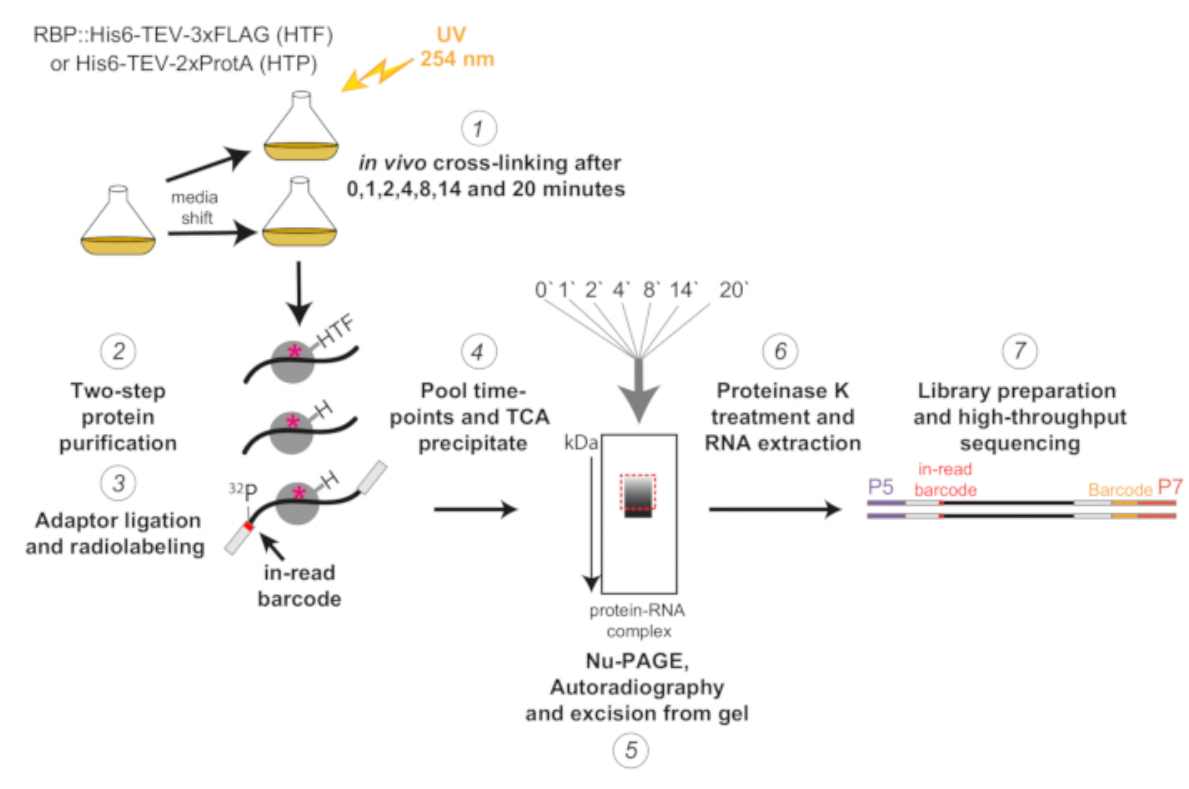

Figure 1: Schematic representation of the $X C R A C$ protocol. Tagged strains were grown until the desired density. RBP indicates RNA-binding protein. Afterwards, a reference sample was taken and cross-linked with $254 \mathrm{~nm}$ UV light. The remaining cells were harvested by filtration and then rapidly shifted to the stress-inducing medium. For the XCRAC experiment described here, samples were taken and cross-linked 1, 2, 4, 8, 14, and 20 min after the shift (1). The RBP of interest was then purified using a highly stringent two-step affinity purification (2). Next, the captured cross-linked RNAs were partially digested with RNases, radiolabeled at the 5' end and adapters were ligated onto them (3). The 5' adapters contained unique "in-read" barcode sequences so that the individual samples could be separated bioinformatically after sequencing. The RBP-RNA complexes were then eluted, pooled, and precipitated together (4), resolved by SDS-PAGE and visualized through autoradiography (5). Subsequently, a single gel slice containing the radioactive signal just above the main band, illustrated with dashed red box in the autoradiography image, was cut from the gel (5). The gel slices were treated with protease $\mathrm{K}$ and the RNA was subsequently extracted (6), converted into cDNAs, and amplified through PCR (7). The PCR step introduced additional barcodes (yellow block introduced by P7 oligo) so that many libraries could be multiplexed into a single lane. Please click here to view a larger version of this figure. 
A

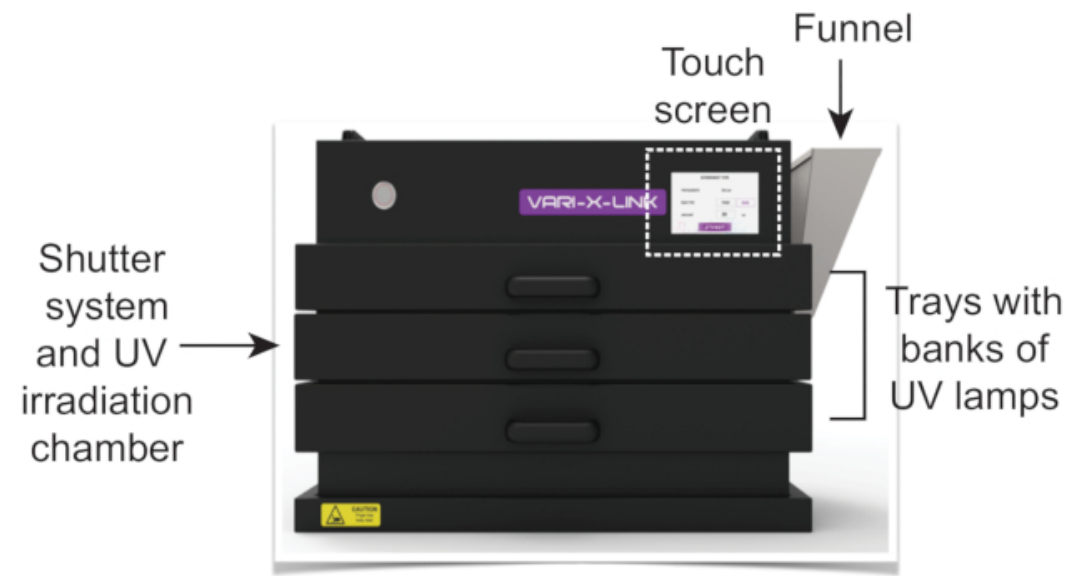

B

Pump for draining cells

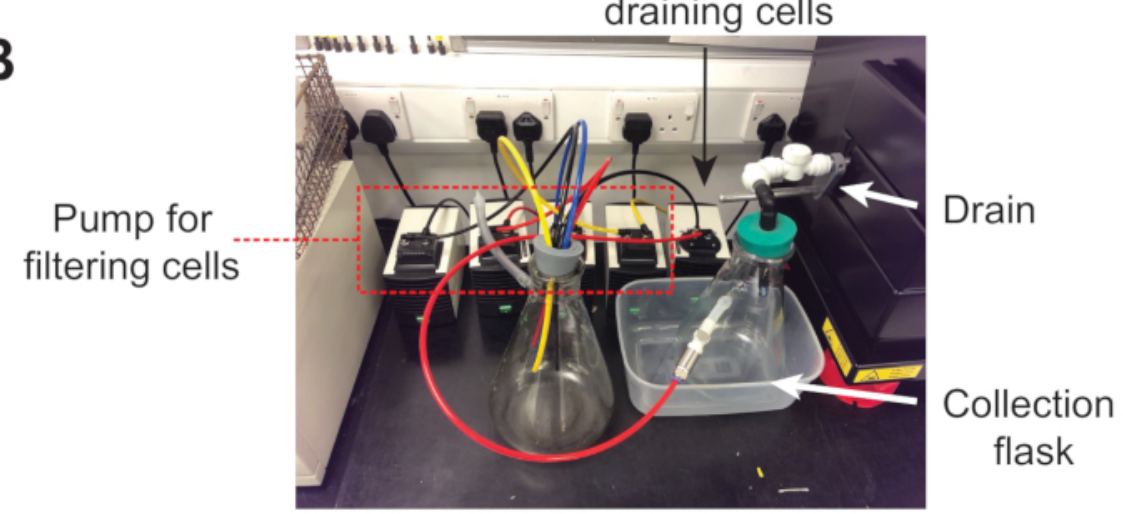

C

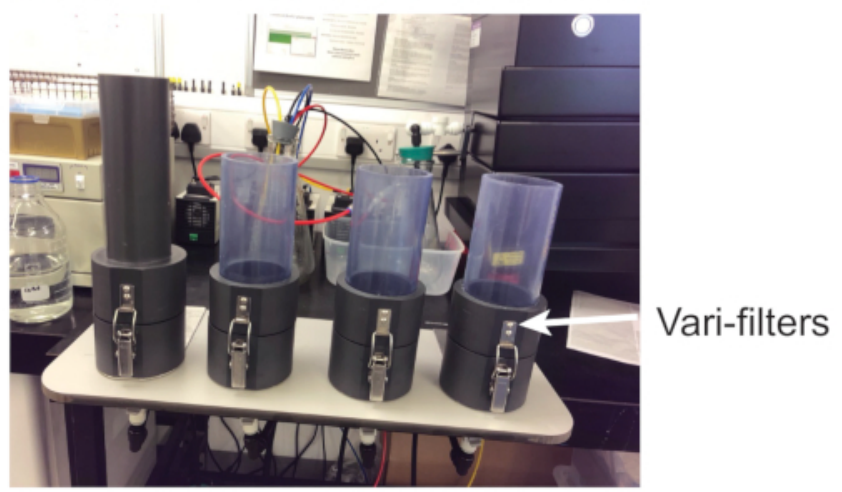

D

valves
below
filters
controlling
the flow

Flasks collecting the flowthrough

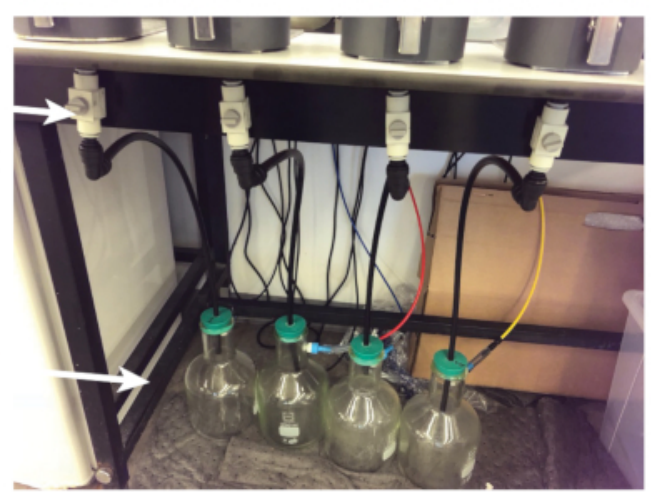

Figure 2: Cross-linking and vacuum filtration. (A) The cross-linker. The cell suspension is poured into a funnel located

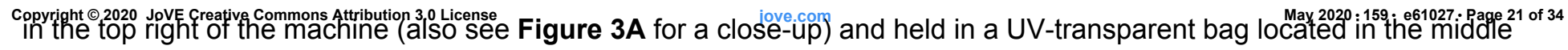



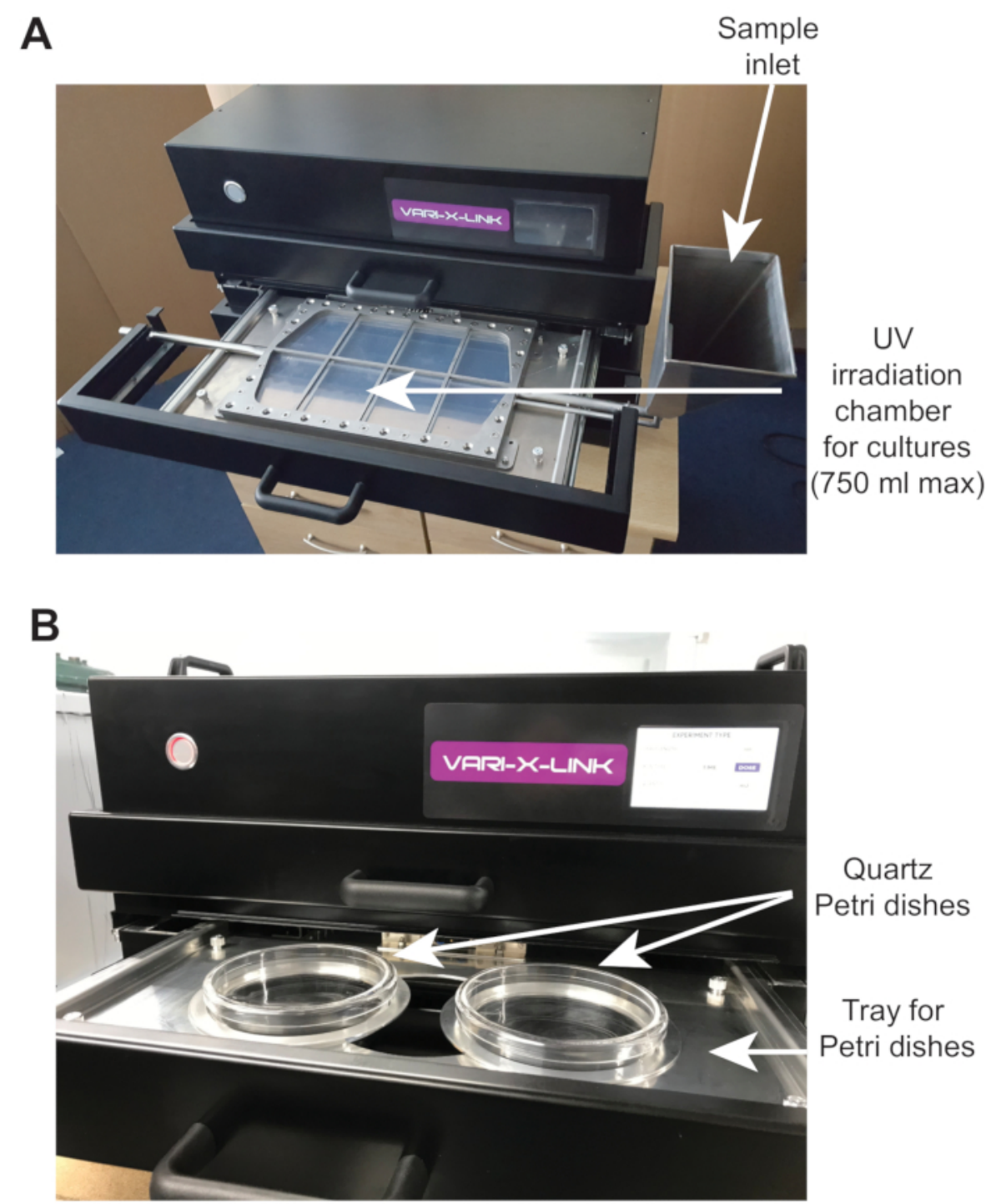

Figure 3: Cross-linking suspended vs. adherent cells. (A) The cross-linker with the Vari-X-linker chamber for suspension cells. The cell culture is poured into the sample inlet (funnel) located in the top right of the tray. (B) Tray that can hold plastic or quartz Petri dishes for cross-linking adherent cells or small volumes of suspension cells. Please click here to view a larger version of this figure. 
A

Time after Autoradiogram B shift (min) $0 \begin{array}{lllllll}0 & 1 & 2 & 4 & 8 & 14 & 20\end{array}$

B Autoradiogram

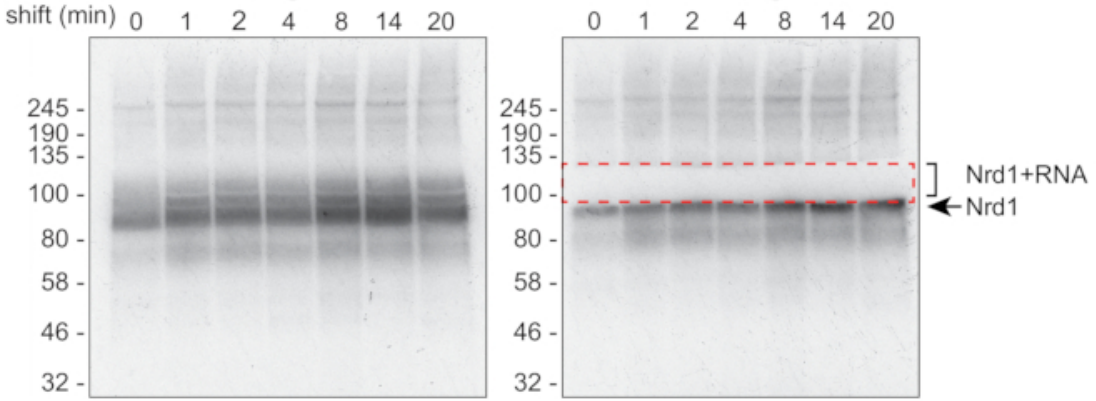

C

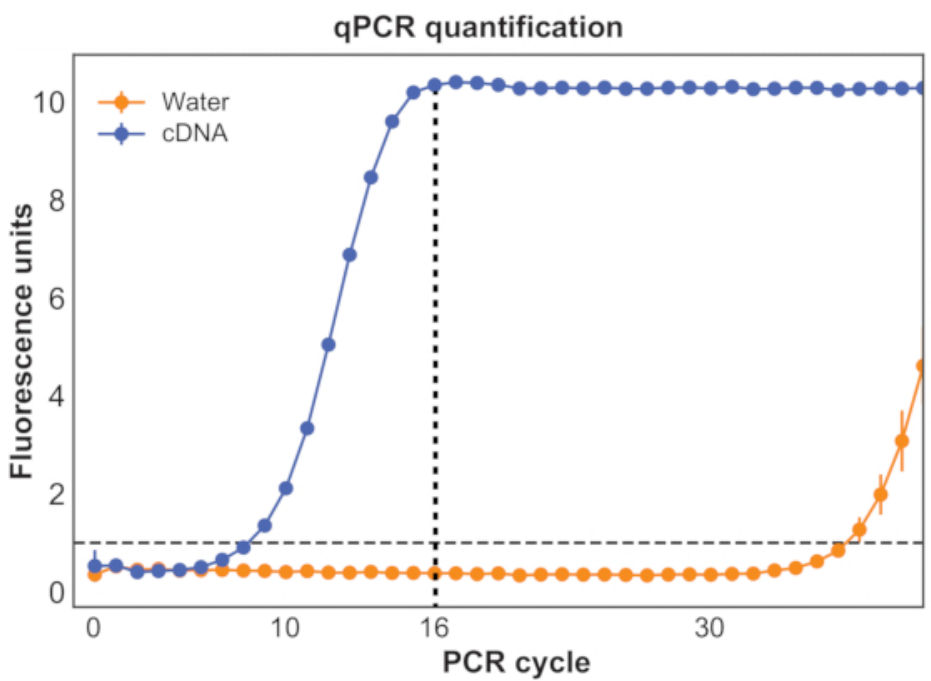

D

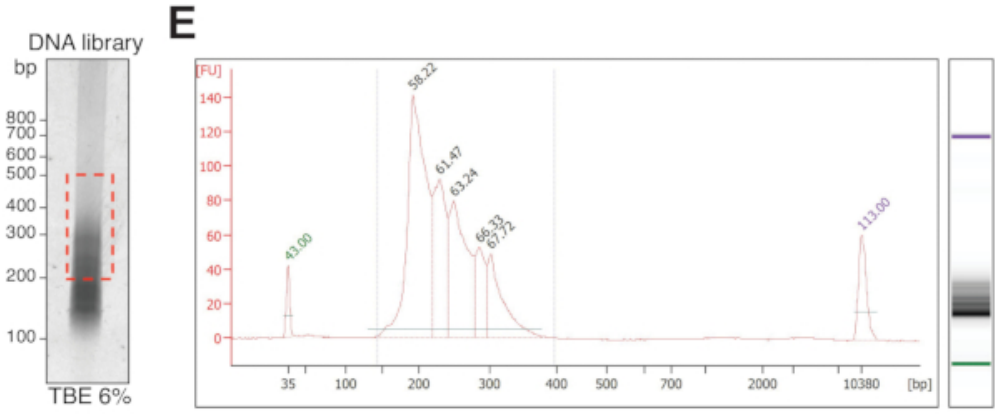

Figure 4: Library preparation. (A) Example of an autoradiogram from a Nrd1-HTP XCRAC experiment. The strong, concentrated signal represents the protein crosslinked to very short RNAs, while the smear above represents the protein cross-linked to RNAs of sufficient length for sequencing. (B) The smear was excised as shown in an autoradiogram taken after gel excision. (C) A representative qPCR from a XCRAC cDNA library. In this example, maximum amplification of the cDNA was reached at 16 cycles. Thus, 16 cycles were used for the final amplification. The error bar represents the standard deviation of three technical qPCR replicates. (D) Example of a phosphorimage from a cDNA library on a $6 \%$ TBE gel. (E) cDNA length and quality analysis from a chip-based capillary electrophoresis. Please click here to view a larger version of this figure. 
A
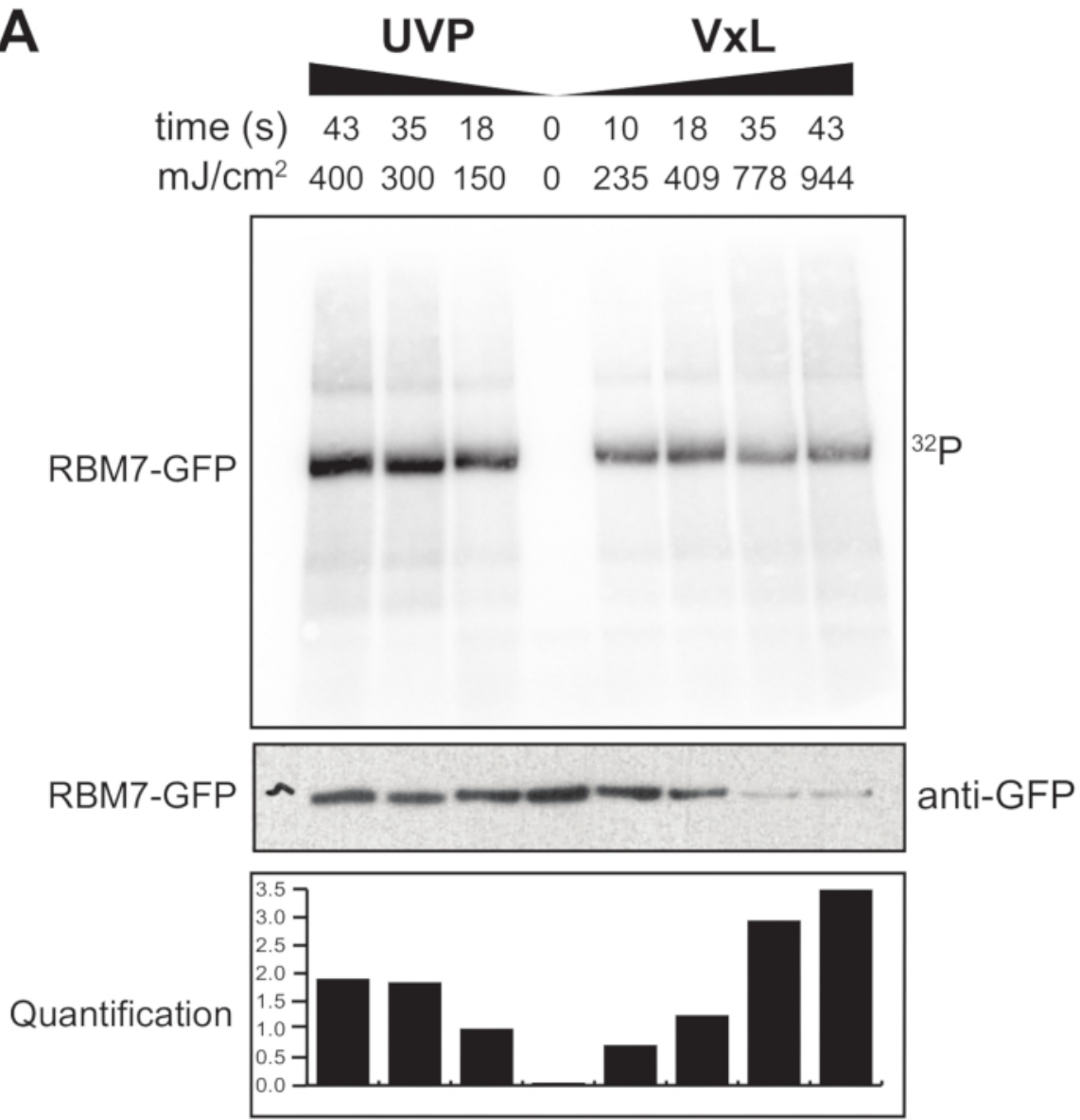

B
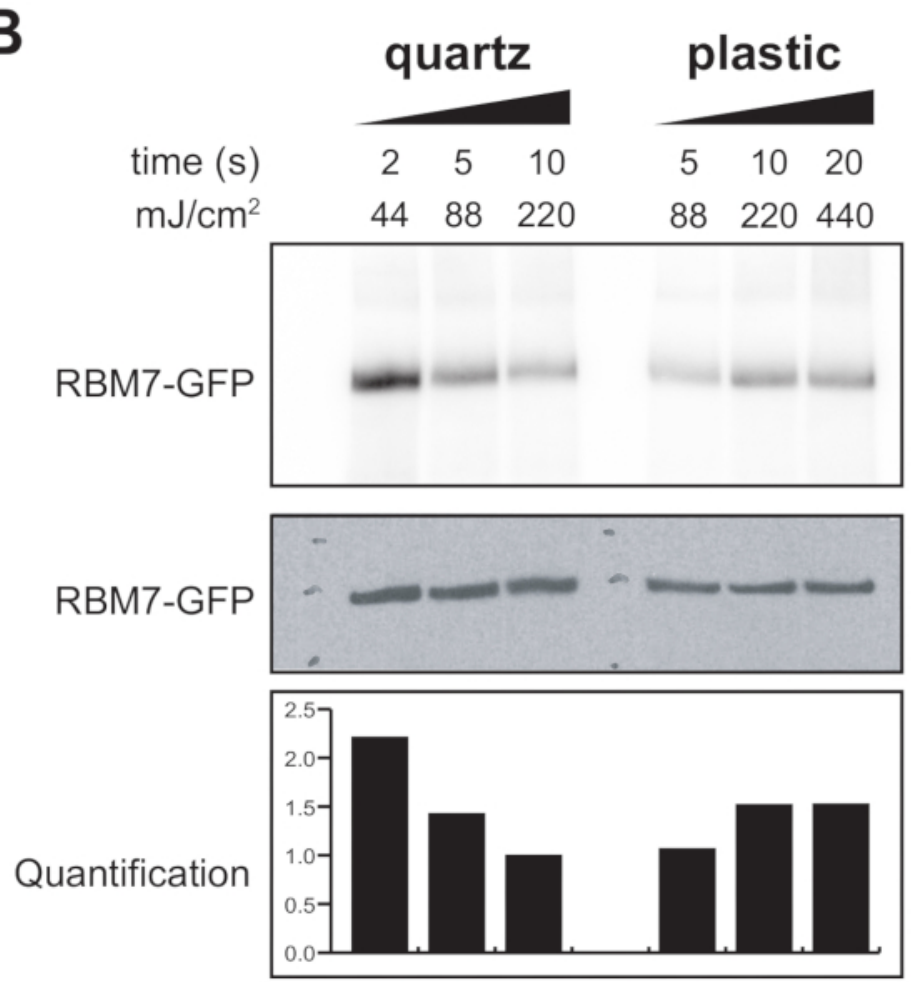

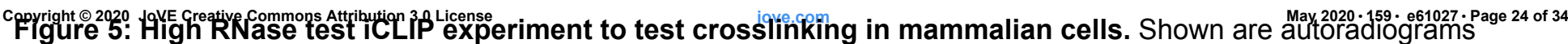


A

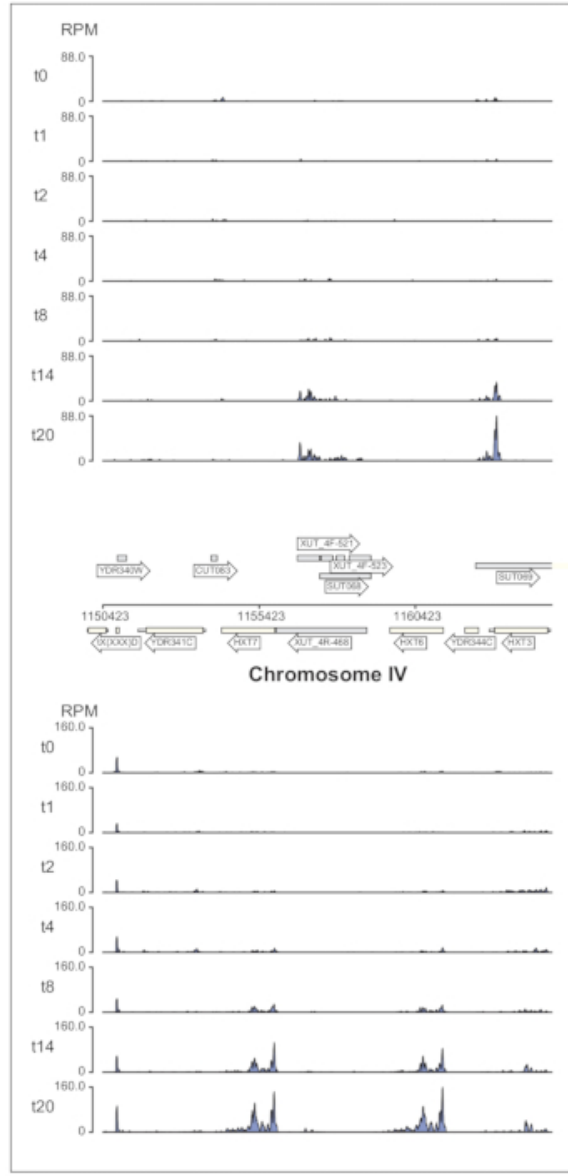

B

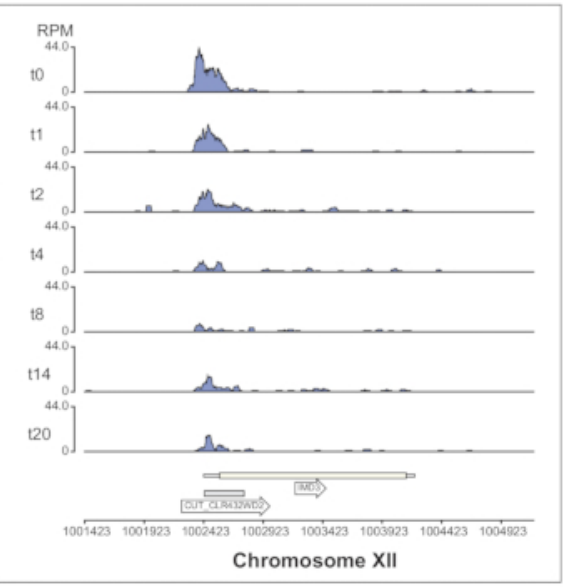

C

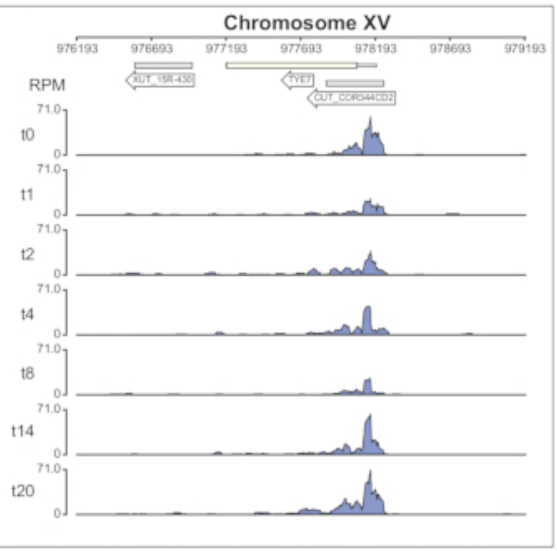

Figure 6: Example genome browser plots showing the power of $X C R A C$ to show differential, temporal binding of Nrd1 to its targets. Each box shows plots for individual genomic regions. The arrows indicate on which strand the genes are encoded (left pointing arrow $=$ minus strand; right pointing arrow $=$ plus strand). The timepoints $(\min )$ are indicated by t0, $\mathrm{t} 1$, $\mathrm{t} 2$, etc on the $\mathrm{y}$-axes of each subplot. Roman numerals indicating the chromosomes and the coordinates are shown. (A) Upon glucose deprivation, Nrd1 binds two high-affinity glucose transporters, HXT6 and HXT7, which are both upregulated in this condition. (B) Nrd1 is observed to bind to Imd3, an already validated target of $\mathrm{Nab3}^{44}$, with reducing intensity following glucose starvation. (C) Nrd1 binding of Tye7 exhibits a dynamic and transient nature, decreasing after glucose starvation to a minimum after 8 min of stress. However, binding subsequently returns to basal levels after 14 min. Reads were normalized to "reads per million" (RPM; y-axis). Grey boxes indicate regions encoding noncoding RNAs. Please click here to view a larger version of this figure. 


\section{Discussion}

The XCRAC method, combined with the new cross-linking and cell harvesting devices, has great potential because it is applicable to a wide range of model organisms and therefore should be of general interest to the RNA field. There are many areas in which $X$ CRAC can be utilized. For example, the method could be used to measure the hierarchical assembly of proteins into large macromolecular complexes, such as the spliceosome and the ribosome, which often involves dynamic interactions between proteins and RNA molecules. We also now routinely use it to monitor interactions between RNA decay factors and their substrates when cells are subjected to diverse kind of stresses. This enables us to determine at what stage of the adaptive response these factors are most active, what substrates they bind to, and how dynamic these interactions are. Such data should enable researchers to determine the relative contribution of each factor in adaptation to environmental changes.

XCRAC uses dual affinity purification tags (HTF or HTP) to purify the protein under highly stringent and denaturing conditions. This ensures that the copurified RNA is highly enriched for RNAs that were covalently cross-linked to the protein of interest. However, relying on affinity tags has disadvantages. For example, the tag could interfere with protein function, which could give a distorted readout of its RNA-binding interactome. Additionally, for some model organisms it may not always be possible to utilize tags because the genetic tools to integrate DNA fragments into the genome or to transform expression plasmids are not yet available. However, it is straightforward to alter some parts of the XCRAC protocol to make it compatible with CLIPbased protocols that rely on antibodies for purification of the RBP. Indeed, this study showed that it is possible to combine iCLIP-based purifications with our crosslinker. We are now 
in the process of developing CLIP protocols to study the temporal association of human RNA-binding proteins with nascent RNA transcripts.

When performing XCRAC on a new protein, the UV exposure must be optimized in order to induce maximal cross-linking. This is important because high UV exposures can reduce the recovery of RNA during the purification step. Cells expressing the recombinant RBP were exposed to various UV doses, 100 $\mathrm{mJ} / \mathrm{cm}^{2}, 250 \mathrm{~mJ} / \mathrm{cm}^{2}, 500 \mathrm{~mJ} / \mathrm{cm}^{2}$, and $1 \mathrm{~J} / \mathrm{cm}^{2}$. The RNPs were then captured and the RNAs were fragmented and radiolabeled. Afterwards, the RNPs were resolved by SDSPAGE and an autoradiogram was taken in order to deduce which exposure gave the most intense signal (i.e. the maximal cross-linking).

Once the experimental conditions are optimized, several control experiments are recommended when performing XCRAC. First, a UV irradiated, untagged sample can be used to monitor background binding to the purification beads. Second, when applying XCRAC during a shift experiment, a second time series where the cells are shifted back into the original medium enables investigation into whether the filtration of the cells itself induces changes in RNA levels or protein-RNA interactions.

As mentioned in the Introduction, numerous recently published papers suggest a number of optimizations to the CLIP protocol. This includes the use of fluorescently labeled adapters for detecting the protein-RNA complex through infrared scanning ${ }^{10}$ as well as optimizations to various nucleic acid purification and size selection steps shown to increase the complexity of the resulting libraries ${ }^{12,45}$. We are currently implementing some of these improvements to further refine the XCRAC protocol. The protocol presented here already contains a number of improvements to the original CRAC 
and $\mathrm{XCRAC}$ protocols that increase the complexity of the data. For example, previously, after resolving the crosslinked, radioactive protein-RNA complexes on SDS-PAGE gels, they were transferred to a nitrocellulose membrane and the cross-linked RNA was isolated from the blot. However, the transfer of the RNP and subsequent RNA extraction can be very inefficient, particularly when dealing with large RBPs such as RNA polymerase subunits. This can result in a significant reduction in the recovery of the crosslinked RNA. In the current protocol, the cross-linked RNA is extracted directly from SDS-PAGE gel slices as illustrated in Figure 1. This increased the recovery of cross-linked RNAs. Additionally, after PCR amplification of the cDNAs the product was originally resolved on $3 \%$, low melting temperature agarose gels, and then 175-300 bp PCR products were extracted from the gel. However, these gels can be easily overloaded, resulting in very poor separation of the DNA. Replacing agarose gels with precast TBE gels resulted in more consistent size separation and better recovery of PCR products.

\section{Disclosures}

A. Langford and W. Worboys are affiliated with UVO3, a commercial company. They had no role in study design, data collection and interpretation, or the decision to submit the work for publication.

\section{Acknowledgments}

This work was supported by grants from the Wellcome Trust (091549 to S.G and 109093/Z/15/A to S.M.), the Wellcome Trust Centre for Cell Biology core grant (092076) and Medical Research Council Non-Clinical Senior Research Fellowship (MR/R008205/1 to S.G.), the European Molecular Biology Organization under a long-term postdoctoral fellowship (ALTF 
1070-2017 to R.A.C), and the Independent Research Fund Denmark (T.H.J).

\section{References}

1. Ule, J. et al. CLIP identifies Nova-regulated RNA networks in the brain. Science. 302 (5648), 1212-1215 (2003).

2. Granneman, S., Kudla, G., Petfalski, E., Tollervey, D. Identification of protein binding sites on U3 snoRNA and pre-rRNA by UV cross-linking and high-throughput analysis of cDNAs. Proceedings of the National Academy of Sciences. 106 (24), 9613-9618 (2009).

3. Licatalosi, D. D. et al. HITS-CLIP yields genome-wide insights into brain alternative RNA processing. Nature. 456 (7221), 464-469 (2008).

4. König, J. et al. iCLIP reveals the function of hnRNP particles in splicing at individual nucleotide resolution. Nature Structural \& Molecular Biology. 17 (7), 909-915 (2010).

5. Hafner, M. et al. Transcriptome-wide Identification of RNA-Binding Protein and MicroRNA Target Sites by PAR-CLIP. Cell. 141 (1), 129-141 (2010).

6. Aktaş, T. et al. DHX9 suppresses RNA processing defects originating from the Alu invasion of the human genome. Nature. 544 (7648), 115-119 (2017).

7. Huppertz, I. et al. iCLIP: Protein-RNA interactions at nucleotide resolution. Methods. 65 (3), 274-287 (2014).

8. $\mathrm{Li}, \mathrm{X}$. et al. Comprehensive in vivo RNA-binding site analyses reveal a role of Prp8 in spliceosomal assembly. Nucleic Acids Research. 41 (6), 3805-3818 (2013).

9. Rosenberg, M. et al. Denaturing CLIP, dCLIP, Pipeline Identifies Discrete RNA Footprints on Chromatin- 
Associated Proteins and Reveals that CBX7 Targets 3' UTRs to Regulate mRNA Expression. Cell Systems. 5 (4), 368-385 (2017).

10. Zarnegar, B.J. et al. irCLIP platform for efficient characterization of protein-RNA interactions. Nature Methods. 13 (6), 489-492 (2016).

11. Kargapolova, Y., Levin, M., Lackner, K., Danckwardt, S. sCLIP-an integrated platform to study RNA-protein interactomes in biomedical research: identification of CSTF2tau in alternative processing of small nuclear RNAs. Nucleic Acids Research. 45 (10), 6074-6086 (2017).

12. Van Nostrand, E. L. et al. Robust transcriptomewide discovery of RNA-binding protein binding sites with enhanced CLIP (eCLIP). Nature Methods. 13 (6), 508-514 (2016).

13. Flynn, R. A. et al. Dissecting noncoding and pathogen RNA-protein interactomes. RNA. 21 (1), 135-143 (2015).

14. Brugiolo, M., Botti, V., Liu, N., Müller-McNicoll, M., Neugebauer, K. M. Fractionation iCLIP detects persistent SR protein binding to conserved, retained introns in chromatin, nucleoplasm and cytoplasm. Nucleic Acids Research. 45 (18), 10452-10465 (2017).

15. Sanford, J. R. et al. Identification of Nuclear and Cytoplasmic mRNA Targets for the Shuttling Protein SF2/ASF. PLOS ONE. 3 (10), e3369 (2008).

16. Garzia, A., Meyer, C., Morozov, P., Sajek, M., Tuschl, T. Optimization of PAR-CLIP for transcriptome-wide identification of binding sites of RNA-binding proteins. Methods. 118-119, 24-40 (2017).

17. Windhager, L. et al. Ultrashort and progressive 4sUtagging reveals key characteristics of RNA processing 
at nucleotide resolution. Genome Research. 22 (10), 2031-2042 (2012).

18. Chen, K. et al. High-Resolution N6-Methyladenosine (m6A) Map Using Photo-Crosslinking-Assisted m6A Sequencing. Angewandte Chemie International Edition. 54 (5), 1587-1590 (2015).

19. $\mathrm{Ke}, \mathrm{S}$. et al. A majority of $\mathrm{m} 6 \mathrm{~A}$ residues are in the last exons, allowing the potential for 3' UTR regulation. Genes \& Development. 29 (19), 2037-2053 (2015).

20. Linder, B. et al. Single-nucleotide-resolution mapping of $\mathrm{m} 6 \mathrm{~A}$ and $\mathrm{m} 6 \mathrm{Am}$ throughout the transcriptome. Nature Methods. 12 (8), 767-772 (2015).

21. Kudla, G., Granneman, S., Hahn, D., Beggs, J. D., Tollervey, D. Cross-linking, ligation, and sequencing of hybrids reveals RNA-RNA interactions in yeast. Proceedings of the National Academy of Sciences. 108 (24), 10010-10015 (2011).

22. Sugimoto, Y. et al. hiCLIP reveals the in vivo atlas of mRNA secondary structures recognized by Staufen 1 . Nature. 519 (7544), 491-494 (2015).

23. Hwang, H.-W. et al. cTag-PAPERCLIP Reveals Alternative Polyadenylation Promotes Cell-Type Specific Protein Diversity and Shifts Araf Isoforms with Microglia Activation. Neuron. 95 (6), 1334-1349.e5 (2017).

24. Hwang, H.-W. et al. PAPERCLIP Identifies MicroRNA Targets and a Role of CstF64/64tau in Promoting Noncanonical poly(A) Site Usage. Cell Reports. 15 (2), 423-435 (2016).

25. Lee, F. C. Y., Ule, J. Advances in CLIP Technologies for Studies of Protein-RNA Interactions. Molecular Cell. 69 (3), 354-369 (2018). 
26. Beckmann, B. M. RNA interactome capture in yeast. Methods. 118-119, 82-92 (2017).

27. Granneman, S., Petfalski, E., Tollervey, D. A cluster of ribosome synthesis factors regulate pre-rRNA folding and 5.8S rRNA maturation by the Rat1 exonuclease. The EMBO Journal. 30 (19), 4006-4019 (2011).

28. Schaughency, P., Merran, J., Corden, J. L. GenomeWide Mapping of Yeast RNA Polymerase II Termination. PLOS Genetics. 10 (10), e1004632 (2014).

29. Bernstein, J. A., Khodursky, A. B., Lin, P.-H., LinChao, S., Cohen, S. N. Global analysis of mRNA decay and abundance in Escherichia coli at single-gene resolution using two-color fluorescent DNA microarrays. Proceedings of the National Academy of Sciences. 99 (15), 9697-9702 (2002).

30. Kresnowati, M. T. A. P. et al. When transcriptome meets metabolome: fast cellular responses of yeast to sudden relief of glucose limitation. Molecular Systems Biology. 2 , 49 (2006).

31. Marguerat, S., Lawler, K., Brazma, A., Bähler, J. Contributions of transcription and mRNA decay to gene expression dynamics of fission yeast in response to oxidative stress. RNA Biology. 11 (6), 702-714 (2014).

32. van Nues, R. et al. Kinetic CRAC uncovers a role for Nab3 in determining gene expression profiles during stress. Nature Communications. 8 (1), 12 (2017).

33. Selinger, D. W., Saxena, R. M., Cheung, K. J., Church, G. M., Rosenow, C. Global RNA Half-Life Analysis in Escherichia coli Reveals Positional Patterns of Transcript Degradation. Genome Research. 13 (2), 216-223 (2003). 
34. Tudek, A., Candelli, T., Libri, D. Non-coding transcription by RNA polymerase II in yeast: Hasard or nécessité? Biochimie. 117, 28-36 (2015).

35. Lingaraju, M. et al. The MTR4 helicase recruits nuclear adaptors of the human RNA exosome using distinct archinteracting motifs. Nature Communications. 10 (1), 1-11 (2019).

36. Lubas, M. et al. Interaction Profiling Identifies the Human Nuclear Exosome Targeting Complex. Molecular Cell. $\mathbf{4 3}$ (4), 624-637 (2011).

37. Conrad, N. K. et al. A yeast heterogeneous nuclear ribonucleoprotein complex associated with RNA polymerase II. Genetics. 154 (2), 557-571 (2000).

38. Darby, M. M., Serebreni, L., Pan, X., Boeke, J. D., Corden, J. L. The Saccharomyces cerevisiae Nrd1-Nab3 Transcription Termination Pathway Acts in Opposition to Ras Signaling and Mediates Response to Nutrient Depletion. Molecular and Cellular Biology. 32 (10), 1762-1775 (2012).

39. Webb, S., Hector, R. D., Kudla, G., Granneman, S. PAR-CLIP data indicate that Nrd1-Nab3-dependent transcription termination regulates expression of hundreds of protein coding genes in yeast. Genome Biology. 15 (1), R8 (2014).

40. Jensen, T. H., Jacquier, A., Libri, D. Dealing with Pervasive Transcription. Molecular Cell. 52 (4), 473-484 (2013).

41. van Dijk, E. L. et al. XUTs are a class of Xrn1-sensitive antisense regulatory non-coding RNA in yeast. Nature. 475 (7354), 114-117 (2011).

42. Thiebaut, M. et al. Futile Cycle of Transcription Initiation and Termination Modulates the Response to Nucleotide 
Shortage in S. cerevisiae. Molecular Cell. 31 (5), 671-682 (2008).

43. Merran, J., Corden, J. L. Yeast RNA-Binding Protein Nab3 Regulates Genes Involved in Nitrogen Metabolism. Molecular and Cellular Biology. 37 (18), pii: e00154-17 (2017).

44. Bresson, S., Tuck, A., Staneva, D., Tollervey, D. Nuclear RNA Decay Pathways Aid Rapid Remodeling of Gene Expression in Yeast. Molecular Cell. 65 (5), 787-800.e5 (2017).

45. Buchbender, A. et al. Improved library preparation with the new iCLIP2 protocol. Methods. In Press (2019). 